\title{
Regularity Estimates for the $p$-Sobolev Flow
}

\author{
Tuomo Kuusi $^{1}$ - Masashi Misawa ${ }^{2}$ Kenta Nakamura ${ }^{3}$ \\ Received: 19 September 2019 / Published online: 25 November 2019 \\ (c) The Author(s) 2019
}

\section{Abstract}

We study doubly nonlinear parabolic equation arising from the gradient flow for $p$ Sobolev type inequality, referred as $p$-Sobolev flow from now on, which includes the classical Yamabe flow on a bounded domain in Euclidean space in the special case $p=2$. In this article we establish a priori estimates and regularity results for the $p$-Sobolev type flow, which are necessary for further analysis and classification of limits as time tends to infinity.

Keywords $p$-Sobolev flow $\cdot$ Regularity $\cdot$ Doubly nonlinear equation

Mathematics Subject Classification 35B45 $\cdot 35 \mathrm{~B} 65 \cdot 35 \mathrm{D} 30 \cdot 35 \mathrm{~K} 61$

\section{Introduction}

Let $\Omega \subset \mathbb{R}^{n}(n \geq 3)$ be a bounded domain with smooth boundary $\partial \Omega$. For any positive $T \leq \infty$, let $\Omega_{T}:=\Omega \times(0, T)$ be the space-time cylinder, and let $\partial_{p} \Omega_{T}$ be the parabolic boundary defined by $(\partial \Omega \times[0, T)) \cup(\Omega \times\{t=0\})$. Throughout the paper we fix $p \in[2, n)$ and set $q:=p^{*}-1$, where $p^{*}:=\frac{n p}{n-p}$ is the Sobolev conjugate of $p$. We consider the following doubly nonlinear parabolic equation:

$\bowtie \quad$ Tuomo Kuusi

tuomo.kuusi@helsinki.fi

Masashi Misawa

mmisawa@kumamoto-u.ac.jp

Kenta Nakamura

k-nakamura@math.kyushu-u.ac.jp

1 Department of Mathematics and Statistics, University of Helsinki, PL 68 (Pietari Kalmin katu 5), 00014 Helsinki, Finland

2 Faculty of Advanced Science and Technology, Kumamoto University, Kumamoto 860-8555, Japan

3 Faculty of Mathematics, Kyushu University, Fukuoka 819-0395, Japan 


$$
\begin{cases}\partial_{t}\left(|u|^{q-1} u\right)-\Delta_{p} u=\lambda(t)|u|^{q-1} u & \text { in } \Omega_{\infty}, \\ u=0 & \text { on } \partial \Omega \times(0, \infty), \\ u(\cdot, 0)=u_{0}(\cdot) & \text { in } \Omega, \\ \|u(t)\|_{L^{q+1}(\Omega)}=1 & \text { for all } t \geq 0 .\end{cases}
$$

Here the unknown function $u=u(x, t)$ is a real-valued function defined for $(x, t) \in$ $\Omega_{\infty}$, and the initial data $u_{0}$ is assumed to be in the Sobolev space $W_{0}^{1, p}(\Omega)$, positive, bounded in $\Omega$ and satisfy $\left\|u_{0}\right\|_{L^{q+1}(\Omega)}=1$, and $\Delta_{p} u:=\operatorname{div}\left(|\nabla u|^{p-2} \nabla u\right)$ is the $p$-Laplacian. The condition imposed in the fourth line of (1.1) is called the volume constraint and $\lambda(t)$ is Lagrange multiplier stemming from this volume constraint. In fact, multiplying (1.1) by $u$ and integrating by parts, we find by a formal computation that $\lambda(t)=\int_{\Omega}|\nabla u(x, t)|^{p} \mathrm{~d} x$ (see Proposition 5.2 below for the rigorous argument). We call the system (1.1) as p-Sobolev flow.

Our main result in this paper is the following theorem.

Theorem 1.1 Let $\Omega$ be a bounded domain with smooth boundary. Suppose that the initial data $u_{0}$ is positive in $\Omega$, belongs to $W_{0}^{1, p}(\Omega) \cap L^{\infty}(\Omega)$, and satisfies the volume constraint $\left\|u_{0}\right\|_{L^{q+1}(\Omega)}=1$. Let $u$ be a weak solution of $(1.1)$ in $\Omega_{\infty} \equiv \Omega \times(0, \infty)$ with the initial and boundary data $u_{0}$. Then, $u$ is positive and bounded in $\Omega_{\infty}$ and, together with its spatial gradient, locally Hölder continuous in $\Omega_{\infty}$. Moreover, for $t \in[0, \infty)$,

$$
\lambda(t)=\int_{\Omega}|\nabla u(x, t)|^{p} \mathrm{~d} x \quad \text { and } \quad \lambda(t) \leq \lambda(0) .
$$

The definition of a weak solution is given in Definition 3.2. The global existence of the $p$-Sobolev flow and its asymptotic behavior, that is the volume concentration at infinite time, will be treated in our forthcoming paper, based on the a-priori regularity estimates for the $p$-Sobolev flow, obtained in the main theorem above (refer to [22,24] for the stationary problem).

The ODE part of the $p$-Sobolev flow equation is of exponential type, since the order of solution in both the time derivative and lower-order terms are the same. Thus, the solution is bounded for all times by the maximum principle (Proposition 3.5). On the other hand, a priori the solution may vanish in a finite time, by the effect of fast diffusion. This undesirable behavior can be ruled out for the $p$-Sobolev flow (1.1) by the volume constraint, that is the preservation of volume at all time. In fact, we show the global positivity of solutions of (1.1) under the volume constraint (Proposition 5.4). The positivity of solutions is based on local energy estimates for truncated solution and De Giorgi's iteration method. For the porous medium and $p$ Laplace equations, see [6,7,27,28], and also [26]. Our task is to discover the intrinsic scaling to the doubly nonlinear operator in the $p$-Sobolev flow (Corollary 4.6). Then, the interior positivity is obtained from some covering argument, being reminiscent of the so-called Harnack chain (Corollary 4.8). This leads to the positivity and regularity on a non-convex domain and thus, may be of interest in geometry. Once the interior positivity is established, the positivity near the boundary of domain follows from the usual comparison function (Proposition 4.9). Finally, the Hölder continuity reduced to 
that of the evolutionary $p$-Laplacian equation, by use of the positivity and boundedness of solutions. The energy equality also holds true for a weak solution of the $p$-Sobolev flow, leading to the continuity on time of the $p$-energy and volume.

The doubly nonlinear equations have been considered by Vespri [29], Porizio and Vespri [20], and Ivanov [11,12]. See also [9,14,16,20,25,30]. The regularity proofs for doubly nonlinear equations are based on the intrinsic scaling method, originally introduced by DiBenedetto, and they have to be arranged in some way depending on the particular form of the equation. Here, the very fast diffusive doubly nonlinear equation such as the $p$-Sobolev flow (1.1) is treated, and the positivity, boundedness and regularity of weak solutions are studied and shown in some precise way. See [19] for existence of a weak solution.

Consider next the stationary equation for (1.1), which is described by the $p$ Laplacian type elliptic equation, obtained from simply removing the time derivative term from the first equation in (1.1). This stationary equation relates to the existence of the extremal function attaining the best constant of Sobolev's embedding inequality, $W_{0}^{1, p}(\Omega) \hookrightarrow L^{q+1}(\Omega)$. If the domain $\Omega$ is star-shaped with respect to the origin, the trivial solution $u \equiv 0$ only exists, by Pohozaev's identity and Hopf's maximum principle. Thus, the $p$-Sobolev flow (1.1), if globally exists, may have finitely many volume concentration points at infinite time. This volume concentration phenomenon is one of our motives of studying the $p$-Sobolev flow (1.1). Moreover, if the domain $\Omega$ is replaced by a smooth compact manifold, we can study the generalization of Yamabe problem in the sense of $p$-Laplacian setting. This is another geometric motive for the $p$-Sobolev flow.

In fact, in the case that $p=2$, our $p$-Sobolev flow (1.1) is exactly the classical Yamabe flow equation in the Euclidean space. The classical Yamabe flow was originally introduced by Hamilton in his study of the so-called Yamabe problem [2,3,31], asking the existence of a conformal metric of constant curvature on $n(\geq 3)$-dimensional closed Riemannian manifolds [10]. Let $\left(\mathcal{M}, g_{0}\right)$ be a $n(\geq 3)$-dimensional smooth, closed Riemannian manifold with scalar curvature $R_{0}=R_{g_{0}}$. The classical Yamabe flow is given by the heat flow equation

$$
u_{t}=(s-R) u=u^{-\frac{4}{n-2}}\left(c_{n} \Delta_{g_{0}} u-R_{0} u\right)+s u,
$$

where $u=u(t), t \geq 0$ is a positive function on $\mathcal{M}$ such that $g(t)=u(t)^{\frac{4}{n-2}} g_{0}$ is a conformal change of a Riemannian metric $g_{0}$, with volume $\operatorname{Vol}(\mathcal{M})=\int_{\mathcal{M}} \operatorname{dvol} g$ $=\int_{\mathcal{M}} u^{\frac{2 n}{n-2}} \mathrm{dvol}_{g_{0}}=1$, having total curvature

$$
s:=\int_{\mathcal{M}}\left(c_{n}|\nabla u|_{g_{0}}^{2}+R_{0} u^{2}\right) \operatorname{dvol}_{g_{0}}=\int_{\mathcal{M}} R \operatorname{dvol}_{g}, \quad c_{n}:=\frac{4(n-1)}{n-2} .
$$

Here we will notice that the condition for volume above naturally corresponds the volume constraint in (1.1). Hamilton [10] proved convergence of the Yamabe flow as $t \rightarrow \infty$ under some geometric conditions. Under the assumption that $\left(\mathcal{M}, g_{0}\right)$ 
is of positive scalar curvature and locally conformal flat, Ye [32] showed the global existence of the Yamabe flow and its convergence as $t \rightarrow \infty$ to a metric of constant scalar curvature. Schwetlick and Struwe [21] succeeded in obtaining the asymptotic convergence of the Yamabe flow in the case $3 \leq n \leq 5$, under an appropriate condition of Yamabe invariance $Y\left(\mathcal{M}, g_{0}\right)$, which is given by infimum of Yamabe energy $E(u)=\int_{\mathcal{M}}\left(c_{n}|\nabla u|_{g_{0}}^{2}+R_{0} u^{2}\right)$ dvol $_{g_{0}}$ among all positive smooth function $u$ on $\mathcal{M}$ with $\operatorname{Vol}(\mathcal{M})=1$, for an initial positive scalar curvature. In Euclidean case, since $R_{g_{0}}=0$ their curvature assumptions are not verified. In outstanding results concerning the Yamabe flow, the equation is equivalently transformed to the scalar curvature equation, and this is crucial for obtaining many properties for the Yamabe flow. In this paper, we are forced to take a direct approach dictated by the structure of the $p$-Laplacian leading to the degenerate/singular parabolic equation of the $p$-Sobolev flow. Let us remark that our results cover those of the classical Yamabe flow in the Euclidian setting.

The structure of this paper is as follows. In Sect. 2 we prepare some notations and technical analysis tools, which are used later. Section 3 provides basic definitions of weak solutions, and also some basic study of the doubly nonlinear equations of $p$ Sobolev flow type, including (1.1), and derivation of the minimum and maximum principles. Moreover, we establish the comparison theorem and make the Caccioppoli type estimates, which have a crucial role in Sect.4. In the next section, Sect.4, we prove the expansion of positivity for the doubly nonlinear equations of $p$-Sobolev flow type. In Sect. 5 we show the positivity, the energy estimates and, consequently, the Hölder regularity for the p-Sobolev flow (1.1). Finally, in Appendices A to C, for the $p$-Sobolev flow, we give detailed proofs of facts used in the previous sections.

\section{Preliminaries}

We prepare some notations and technical analysis tools, which are used later.

\subsection{Notation}

Let $\Omega$ be a bounded domain in $\mathbb{R}^{n}(n \geq 3)$ with smooth boundary $\partial \Omega$ and for a positive $T \leq \infty$ let $\Omega_{T}:=\Omega \times(0, T)$ be the cylindrical domain. Let us define the parabolic boundary of $\Omega_{T}$ by

$$
\partial_{p} \Omega_{T}:=\partial \Omega \times[0, T) \cup \Omega \times\{t=0\} .
$$

We prepare some function spaces, defined on space-time region. For $1 \leq p, q \leq \infty$, $L^{q}\left(t_{1}, t_{2} ; L^{p}(\Omega)\right)$ is a function space of measurable real-valued functions on a spacetime region $\Omega \times\left(t_{1}, t_{2}\right)$ with a finite norm

$$
\|v\|_{L^{q}\left(t_{1}, t_{2} ; L^{p}(\Omega)\right)}:= \begin{cases}\left(\int_{t_{1}}^{t_{2}}\|v(t)\|_{L^{p}(\Omega)}^{q} \mathrm{~d} t\right)^{1 / q} & (1 \leq q<\infty), \\ {\operatorname{ess} \sup _{1}\|v(t)\|_{L^{p}(\Omega)}}_{t_{1} \leq t_{2}} & (q=\infty),\end{cases}
$$


where

$$
\|v(t)\|_{L^{p}(\Omega)}:= \begin{cases}\left(\int_{\Omega}|v(x, t)|^{p} \mathrm{~d} x\right)^{1 / p} & (1 \leq p<\infty), \\ \underset{x \in \Omega}{\operatorname{ess} \sup |v(x, t)|} & (p=\infty) .\end{cases}
$$

When $p=q$, we write $L^{p}\left(\Omega \times\left(t_{1}, t_{2}\right)\right)=L^{p}\left(t_{1}, t_{2} ; L^{p}(\Omega)\right)$ for brevity. For $1 \leq$ $p<\infty$ the Sobolev space $W^{1, p}(\Omega)$ consists of measurable real-valued functions that are weakly differentiable and their weak derivatives are $p$ th integrable on $\Omega$, with the norm

$$
\|v\|_{W^{1, p}(\Omega)}:=\left(\int_{\Omega}|v|^{p}+|\nabla v|^{p} \mathrm{~d} x\right)^{1 / p},
$$

where $\nabla v=\left(v_{x_{1}}, \ldots, v_{x_{n}}\right)$ denotes the gradient of $v$ in a distribution sense, and let $W_{0}^{1, p}(\Omega)$ be the closure of $C_{0}^{\infty}(\Omega)$ with resptect to the norm $\|\cdot\|_{W^{1, p}}$. Also let $L^{q}\left(t_{1}, t_{2} ; W_{0}^{1, p}(\Omega)\right)$ denote a function space of measurable real-valued functions on space-time region with a finite norm

$$
\|v\|_{L^{q}\left(t_{1}, t_{2} ; W_{0}^{1, p}(\Omega)\right)}:=\left(\int_{t_{1}}^{t_{2}}\|v(t)\|_{W^{1, p}(\Omega)}^{q} \mathrm{~d} t\right)^{1 / q} .
$$

Let $B=B_{\rho}\left(x_{0}\right):=\left\{x \in \mathbb{R}^{n}:\left|x-x_{0}\right|<\rho\right\}$ denote an open ball with radius $\rho>0$ centered at some $x_{0} \in \mathbb{R}^{n}$. Let $E \subset \mathbb{R}^{n}$ be a bounded domain. For a real number $k$ and for a function $v$ in $L^{1}(E)$ we define the truncation of $v$ by

$$
(v-k)_{+}:=\max \{(v-k), 0\} ; \quad(k-v)_{+}:=\max \{(k-v), 0\} .
$$

For a measurable function $v$ in $L^{1}(E)$ and a pair of real numbers $k<l$, we set

$$
\left\{\begin{array}{l}
E \cap\{v>l\}:=\{x \in E: v(x)>l\}, \\
E \cap\{v<k\}:=\{x \in E: v(x)<k\}, \\
E \cap\{k<v<l\}:=\{x \in E: k<v(x)<l\} .
\end{array}\right.
$$

Let $z=(x, t) \in \mathbb{R}^{n} \times \mathbb{R}$ be a space-time variable and $\mathrm{d} z=\mathrm{d} x \mathrm{~d} t$ be the space-time volume element.

\subsection{Technical Tools}

We first recall the following De Giorgi's inequality (see [6]).

Proposition 2.1 (De Giorgi's inequality) Let $v \in W^{1,1}(B)$ and $k, l \in \mathbb{R}$ satisfying $k<l$. Then there exists a positive constant $C$ depending only on $p, n$ such that

$$
(l-k)|B \cap\{v>l\}| \leq C \frac{\rho^{n+1}}{|B \cap\{v<k\}|} \int_{B \cap\{k<v<l\}}|\nabla v| \mathrm{d} x .
$$


Let $q=n p /(n-p)-1$ as before. Following [6], we define the auxiliary function

$$
\left\{\begin{array}{l}
A^{+}(k, u):=\int_{k^{q}}^{u^{q}}\left(\xi^{1 / q}-k\right)_{+} \mathrm{d} \xi \\
A^{-}(k, u):=\int_{u^{q}}^{k^{q}}\left(k-\xi^{1 / q}\right)_{+} \mathrm{d} \xi
\end{array}\right.
$$

for $u \geq 0$ and $k \geq 0$. Changing a variable $\eta=\xi^{1 / q}$, we have

$$
A^{+}(k, u)=q \int_{k}^{u}(\eta-k)_{+} \eta^{q-1} \mathrm{~d} \eta=q \int_{0}^{(u-k)_{+}}(\eta+k)^{q-1} \eta \mathrm{d} \eta
$$

and

$$
A^{-}(k, u)=q \int_{u}^{k}(k-\eta)_{+} \eta^{q-1} \mathrm{~d} \eta=q \int_{0}^{(k-u)_{+}}(k-\eta)^{q-1} \eta \mathrm{d} \eta .
$$

Then we formally get

$$
\frac{\partial}{\partial t} A^{+}(k, u)=\frac{\partial u^{q}}{\partial t}(u-k)_{+}
$$

and

$$
\frac{\partial}{\partial t} A^{-}(k, u)=-\frac{\partial u^{q}}{\partial t}(k-u)_{+} .
$$

If $k=0$, we abbreviate as

$$
A^{+}(u)=A^{+}(0, u) \text { and } A^{-}(u)=A^{-}(0, u) .
$$

Let $0<t_{1}<t_{2} \leq T$ and let $K$ be any domain in $\Omega$. We denote a parabolic cylinder by $K_{t_{1}, t_{2}}:=K \times\left(t_{1}, t_{2}\right)$. We recall the Sobolev embedding of parabolic type.

Proposition 2.2 [6] There exists a constant $C$ depending only on $n, p, r$ such that for every $v \in L^{\infty}\left(t_{1}, t_{2} ; L^{r}(K)\right) \cap L^{p}\left(t_{1}, t_{2} ; W_{0}^{1, p}(K)\right)$

$$
\int_{K_{t_{1}, t_{2}}}|v|^{p \frac{n+r}{n}} \mathrm{~d} z \leq C\left(\int_{K_{t_{1}, t_{2}}}|\nabla v|^{p} \mathrm{~d} z\right)\left(\underset{t_{1}<t<t_{2}}{\operatorname{ess} \sup } \int_{\Omega}|v|^{r} \mathrm{~d} x\right)^{\frac{p}{n}}
$$

We next use so-called fast geometric convergence which will be employed later on many times. See [6] for details.

Lemma 2.3 (Fast geometric convergence, [6]) Let $\left\{Y_{m}\right\}_{m=0}^{\infty}$ be a sequence of positive numbers, satisfying the recursive inequlities

$$
Y_{m+1} \leq C b^{m} Y_{m}^{1+\alpha}, \quad m=0,1, \ldots,
$$

where $C, b>1$ and $\alpha>0$ are given constants independent of $m$. If the initial value $Y_{0}$ satisfies

$$
Y_{0} \leq C^{-1 / \alpha} b^{-1 / \alpha^{2}}
$$


then $\lim _{m \rightarrow \infty} Y_{m}=0$.

We also need a fundamental algebraic inequality, associated with the $p$-Laplace operator (see [5]).

Lemma 2.4 For all $p \in(1, \infty)$ there exist positive constants $C_{1}(p, n)$ and $C_{2}(p, n)$ such that for all $\xi, \eta \in \mathbb{R}^{n}$

$$
\left.|| \xi\right|^{p-2} \xi-|\eta|^{p-2} \eta\left|\leq C_{1}(|\xi|+|\eta|)^{p-2}\right| \xi-\eta \mid
$$

and

$$
\left(|\xi|^{p-2} \xi-|\eta|^{p-2} \eta\right) \cdot(\xi-\eta) \geq C_{2}(|\xi|+|\eta|)^{p-2}|\xi-\eta|^{2},
$$

where dot $\cdot$ denotes the inner product in $\mathbb{R}^{n}$. In particular, if $p \geq 2$, then

$$
\left(|\xi|^{p-2} \xi-|\eta|^{p-2} \eta\right) \cdot(\xi-\eta) \geq C_{2}|\xi-\eta|^{p} .
$$

\section{Doubly Nonlinear Equations of $p$-Sobolev Flow Type}

Let $T \leq \infty$. We study the following a doubly nonlinear equation of $p$-Sobolev flow type:

$$
\left\{\begin{array}{l}
\partial_{t}\left(|u|^{q-1} u\right)-\Delta_{p} u=c|u|^{q-1} u \text { in } \Omega_{T} \\
0 \leq u \leq M \text { on } \partial_{p} \Omega_{T}
\end{array}\right.
$$

where $u=u(x, t): \Omega_{T} \rightarrow \mathbb{R}$ be unknown real valued function, and $c$ and $M$ are nonnegative constant and positive one, respectively. This section is devoted to some a priori estimates of a weak solution to (3.1). Firstly, we recall the definition of weak solution of (3.1).

Definition 3.1 A measurable function $u$ defined on $\Omega_{T}$ is called a weak supersolution (subsolution) to (3.1) if the following (D1)-(D3) are satisfied:

(D1) $u \in L^{\infty}\left(0, T ; W^{1, p}(\Omega)\right) ; \partial_{t}\left(|u|^{q-1} u\right) \in L^{2}\left(\Omega_{T}\right)$.

(D2) For every nonnegative $\varphi \in C_{0}^{\infty}\left(\Omega_{T}\right)$,

$$
-\int_{\Omega_{T}}|u|^{q-1} u \varphi_{t} \mathrm{~d} z+\int_{\Omega_{T}}|\nabla u|^{p-2} \nabla u \cdot \nabla \varphi \mathrm{d} z \geq(\leq) c \int_{\Omega_{T}}|u|^{q-1} u \varphi \mathrm{d} z .
$$

(D3) $0 \leq u \leq M$ on $\partial_{p} \Omega_{T}$ in the trace sense:

$(-u(t))_{+},\left((u(t))_{+}-M\right)_{+} \in W_{0}^{1, p}(\Omega)$ for almost every $t \in(0, T) ;$

$$
\int_{\Omega}(-u(x, t))_{+}^{q+1} \mathrm{~d} x, \quad \int_{\Omega}\left((u(x, t))_{+}-M\right)_{+}^{q+1} \mathrm{~d} x \rightarrow 0 \text { as } t \searrow 0 .
$$

A measurable function $u$ defined on $\Omega \times[0, T]$ is called a weak solution to (3.1) if it is simultaneously a weak sub and supersolution; that is,

$$
-\int_{\Omega_{T}}|u|^{q-1} u \varphi_{t} \mathrm{~d} z+\int_{\Omega_{T}}|\nabla u|^{p-2} \nabla u \cdot \nabla \varphi \mathrm{d} z=c \int_{\Omega_{T}}|u|^{q-1} u \varphi \mathrm{d} z
$$


for every $\varphi \in C_{0}^{\infty}\left(\Omega_{T}\right)$.

Similarly,

Definition 3.2 A measurable function $u$ defined on $\Omega_{T}$ is called a weak solution of (1.1) if the following (D1)-(D4) are satisfied:

(D1) $u \in L^{\infty}\left(0, T ; W^{1, p}(\Omega)\right) ; \quad \partial_{t}\left(|u|^{q-1} u\right) \in L^{2}\left(\Omega_{T}\right)$.

(D2) There exists a function $\lambda(t) \in L^{1}(0, T)$ such that, for every $\varphi \in C_{0}^{\infty}\left(\Omega_{T}\right)$,

$$
-\int_{\Omega_{T}}|u|^{q-1} u \varphi_{t} \mathrm{~d} z+\int_{\Omega_{T}}|\nabla u|^{p-2} \nabla u \cdot \nabla \varphi \mathrm{d} z=\int_{\Omega_{T}} \lambda(t)|u|^{q-1} u \varphi \mathrm{d} z .
$$

(D3) $\|u(t)\|_{L^{q+1}(\Omega)}=1$ for all $t \geq 0$.

(D4) $u(0)=u_{0}$ in $\Omega$ and $u=0$ on $\partial_{p} \Omega \times(0, T)$ in the trace sense:

$u(t) \in W_{0}^{1, p}(\Omega)$ for almost every $t \in(0, T)$;

$$
\left\|u(t)-u_{0}\right\|_{\left.L^{q+1} \Omega\right)} \rightarrow 0 \text { as } t \searrow 0 .
$$

Remark 3.3 A solution of our $p$-Sobolev flow equation (1.1) is a subsolution of (3.1) with $c=\left\|\nabla u_{0}\right\|_{L^{p}(\Omega)}^{p}$ and a supersolution of (3.1) with $c=0$, respectively. See the energy estimate (5.3) in Proposition 5.2 below.

\subsection{Nonnegativity and Boundedness}

We next claim that a weak supersolutions to (3.1) are nonnegative, i.e., they satisfy the weak minimum principle.

Proposition 3.4 (Nonnegativity) A weak supersolution u to (3.1) satisfies

$$
u \geq 0 \text { in } \Omega_{T} \text {. }
$$

Proof If $u$ is a weak supersolution to (3.1), $-u$ is a weak subsolution. We note by (D1) in Definition 3.1 that $\partial_{t}\left(|u|^{q-1} u\right) \in L^{2}\left(\Omega_{T}\right)$ and that $(-u)_{+} \in$ $L^{\infty}\left(0, T ; W_{0}^{1, p}(\Omega)\right) \subset L^{q+1}\left(\Omega_{T}\right) \subset L^{2}\left(\Omega_{T}\right)$. Let $0<t_{1}<t \leq T$ be arbitrarily taken and fixed. Put $\Omega_{t_{1}, t}=\Omega \times\left(t_{1}, t\right)$. Let $\delta$ be any positive number such that $\delta \leq\left(t-t_{1}\right) / 3$. We define a Lipschitz cut-off function on time, $\sigma_{t_{1}, t}$ such that

$$
0 \leq \sigma_{t_{1}, t} \leq 1, \quad \sigma_{t_{1}, t}=1 \quad \text { in } \quad\left(t_{1}+\delta, t-\delta\right) \quad \text { and } \operatorname{supp}\left(\sigma_{t_{1}, t}\right) \subset\left(t_{1}, t\right)
$$

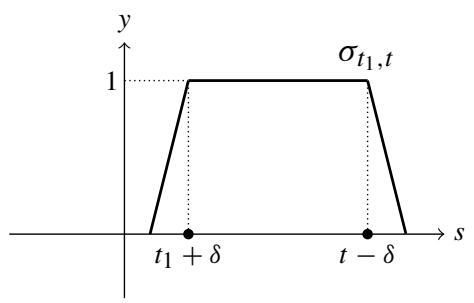


A function $(-u)_{+} \sigma_{t_{1}, t}$ is an admissible test function in (D2) of Definition 3.1. From (D2) of Definition 3.1 we obtain that

$$
\begin{aligned}
& \int_{\Omega_{t_{1}, t}} \partial_{t}\left(|u|^{q-1}(-u)\right)(-u)_{+} \sigma_{t_{1}, t} \mathrm{~d} z+\int_{\Omega_{t_{1}, t}}|\nabla u|^{p-2} \nabla(-u) \cdot \nabla\left((-u)_{+} \sigma_{t_{1}, t}\right) \mathrm{d} z \\
& \quad \leq c \int_{\Omega_{t_{1}, t}}|u|^{q-1}(-u)(-u)_{+} \sigma_{t_{1}, t} \mathrm{~d} z .
\end{aligned}
$$

The first integral on the left hand side of (3.3) is computed as

$$
\begin{aligned}
\int_{\Omega_{t_{1}, t}} \partial_{t}\left(|u|^{q-1}(-u)\right)(-u)_{+} \sigma_{t_{1}, t} \mathrm{~d} z= & \int_{\Omega_{t_{1}, t}} \partial_{t} A^{+}\left((-u)_{+}\right) \sigma_{t_{1}, t} \mathrm{~d} z \\
= & \left.\int_{\Omega} A^{+}\left((-u)_{+} \sigma_{t_{1}, t}\right) \mathrm{d} x\right|_{t_{1}} ^{t} \\
& -\int_{\Omega_{t_{1}, t}} A^{+}\left((-u)_{+}\right) \partial_{t} \sigma_{t_{1}, t} \mathrm{~d} z \\
= & \frac{1}{\delta}\left(\int_{t-\delta}^{t}-\int_{t_{1}}^{t_{1}+\delta}\right) \int_{\Omega} A^{+}\left((-u(s))_{+}\right) \mathrm{d} x \mathrm{~d} s
\end{aligned}
$$

which, as $\delta \rightarrow 0$, converges to

$$
\begin{aligned}
\int_{\Omega} A^{+} & \left((-u(t))_{+}\right) \mathrm{d} x-\int_{\Omega} A^{+}\left(\left(-u\left(t_{1}\right)\right)_{+}\right) \mathrm{d} x \\
& =\frac{q}{q+1} \int_{\Omega}(-u(t))_{+}^{q+1} \mathrm{~d} x-\frac{q}{q+1} \int_{\Omega}\left(-u\left(t_{1}\right)\right)_{+}^{q+1} \mathrm{~d} x
\end{aligned}
$$

where by (D3) in Definition 3.1 we have that

$$
\lim _{t_{1} \rightarrow 0} \int_{\Omega}\left(-u\left(t_{1}\right)\right)_{+}^{q+1} \mathrm{~d} x \rightarrow 0 \text { as } t_{1} \rightarrow 0
$$

The second integral on the left hand side of (3.3) is bounded from below as

$$
\int_{\Omega_{t}}\left|\nabla(-u)_{+}\right|^{p} \sigma_{t_{1}, t} \mathrm{~d} z \geq 0
$$

Taking the limit in (3.3) as $\delta \searrow 0$ and $t_{1} \searrow 0$, and combining (3.4), (3.5), (3.6) with (3.7), we get

$$
\frac{q}{q+1} \int_{\Omega}(-u(t))_{+}^{q+1} \mathrm{~d} x \leq c \int_{0}^{t} \int_{\Omega}(-u(\tau))_{+}^{q+1} \mathrm{~d} x \mathrm{~d} \tau
$$


and, by Gronwall's lemma,

$$
\int_{\Omega}(-u(t))_{+}^{q+1} \mathrm{~d} x \leq 0
$$

since again, by (D3) of Definition 3.1, $(-u(t))_{+} \rightarrow 0$ in $L^{q+1}(\Omega)$ as $t \searrow 0$. Thus we have $-u(x, t) \leq 0$ for $(x, t) \in \Omega_{T}$ and the claim is verified.

We next show the boundedness of the solution.

Proposition 3.5 (Boundedness) Let $u$ be a weak subsolution of (3.1) such that $(u(t))_{+} \in W_{0}^{1, p}(\Omega)$ for almost every $t \in(0, T)$. Then

$$
\left\|(u(t))_{+}\right\|_{L^{\infty}(\Omega)} \leq e^{c T / q}\left\|u_{0}\right\|_{L^{\infty}(\Omega)} .
$$

Proof We will follow the similar argument as in [1]. Set $M:=\left\|u_{0}\right\|_{L^{\infty}(\Omega)}$, so that $0 \leq u \leq M$ on $\partial_{p} \Omega_{T}$. Let us define, for a small $\delta>0$, the Lipschitz truncated function $\phi_{\delta}(u)$ by

$$
\phi_{\delta}(u):=\min \left\{1, \frac{\left(e^{-c t / q} u-M\right)_{+}}{\delta}\right\}
$$

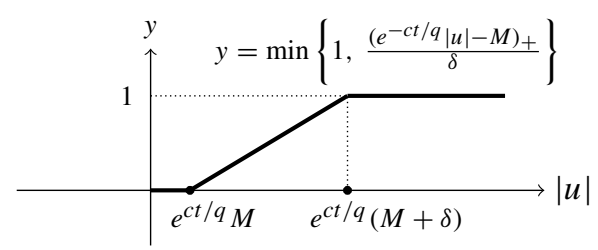

where we note that the support of $\phi_{\delta}$ is $\left\{u>e^{c t / q} M\right\}$, and $\phi_{\delta}(u), \phi_{\delta}^{\prime}(u) \in L^{\infty}\left(\Omega_{T}\right)$, $\phi_{\delta}(0)=0$, and further $\phi_{\delta}(u) \in L^{\infty}\left(0, T ; W_{0}^{1, p}(\Omega)\right)$. Let $0<t_{1}<t \leq T$ and $\sigma_{t_{1}, t}$ be the same time cut-off function as in the proof of Proposition 3.4. The function $e^{-c t} \sigma_{t_{1}, t} \phi_{\delta}(u)$ is an admissible test function in (D2) in Definition 3.1. Choose a test function as $e^{-c t} \sigma_{t_{1}, t} \phi_{\delta}(u)$ in (D2) in Definition 3.1 to have

$$
\int_{\Omega_{t_{1}, t}} \partial_{t}\left(e^{-c t}|u|^{q-1} u\right) \sigma_{t_{1}, t} \phi_{\delta}(u) \mathrm{d} z+\int_{\Omega_{t_{1}, t}}|\nabla u|^{p-2} \nabla u \cdot \nabla\left(e^{-c t} \sigma_{t_{1}, t} \phi_{\delta}(u)\right) \mathrm{d} z \leq 0 .
$$

The first term on the left of (3.9) is computed as

$$
\int_{\Omega_{t_{1}, t}} \partial_{t}\left(e^{-c t}|u|^{q-1} u\right) \min \left\{1, \frac{\left(e^{-c t / q} u-M\right)_{+}}{\delta}\right\} \sigma_{t_{1}, t} \mathrm{~d} z
$$

Since, on the support of $\phi_{\delta},\left\{u>e^{c t / q} M\right\}$,

$$
\nabla u \cdot \nabla \phi_{\delta}(u)=\frac{1}{\delta} \chi_{\left\{e^{c t / q} M<u \leq e^{c t / q}(M+\delta)\right\}}|\nabla u|^{2},
$$


the second term is estimated as

$$
\frac{|\nabla u|^{p}}{\delta} \chi_{\left\{e^{c t / q} M<u \leq e^{c t / q}(M+\delta)\right\}} \sigma_{t_{1}, t} e^{-c t} \geq 0
$$

Gathering (3.9), (3.10) and (3.11), we obtain

$$
\int_{\Omega_{t_{1}, t}} \partial_{t}\left(e^{-c t}|u|^{q-1} u\right) \min \left\{1, \frac{\left(e^{-c t / q} u-M\right)_{+}}{\delta}\right\} \sigma_{t_{1}, t} \mathrm{~d} z \leq 0 .
$$

Since $\partial_{t}\left(|u|^{q-1} u\right)=\partial_{t} u^{q} \in L^{2}(\Omega)$ in $\left\{(u)_{+}>0\right\}$ by (D1) of Definition 3.1, it holds that $\partial_{t}\left(e^{-c t}(u)_{+}^{q}\right) \in L^{2}\left(\Omega_{T}\right)$. Taking the limit as $\delta \searrow 0$ in (3.12), by the Lebesgue dominated convergence theorem, we have that

$$
\int_{\Omega_{t_{1}, t}} \partial_{t}\left(e^{-c t} u^{q}\right) \chi_{\left\{u>e^{c t / q} M\right\}} \mathrm{d} z \leq 0
$$

namely,

$$
\int_{\Omega_{t_{1}, t}} \partial_{t}\left(e^{-c t} u^{q}-M^{q}\right)_{+} \mathrm{d} x \mathrm{~d} t \leq 0 .
$$

By (D3) in Definition 3.1

$$
\int_{\Omega}\left(e^{-c t_{1}}\left(u\left(t_{1}\right)\right)_{+}^{q}-M^{q}\right)_{+} \mathrm{d} x \leq \int_{\Omega}\left(\left(u\left(t_{1}\right)\right)_{+}^{q}-M^{q}\right)_{+} \mathrm{d} x \rightarrow 0
$$

as $t_{1} \searrow 0$. Hence, we pass to the limit as $t_{1} \searrow 0$ in (3.13) to have

$$
\int_{\Omega}\left(e^{-c t}(u(t))^{q}-M^{q}\right)_{+} \mathrm{d} x \leq 0
$$

if and only if $(u)_{+} \leq e^{c t / q} M$ in $\Omega \times[0, T]$, and we arrive at the assertion.

\subsection{Comparison Theorem}

We recall the crucial fact, addressed by Alt and Luckhaus [1], the Comparison theorem [1, Theorem 2.2, p. 325]. For stating it without loss of generality, we define a weak sub and super solutions. A measurable function $u$ and $v$ on $\Omega_{T}$ are a weak supersolution and subsolution, respectively, if the conditions (D1) and (D2) in Definition 3.1 are satisfied. We say that $u \geq v$ on $\partial_{p} \Omega_{T}$ in the trace sense, if

$$
\begin{aligned}
\text { (D3') } & (-u(t)+v(t))_{+} \in W_{0}^{1, p}(\Omega), \text { for almost every } t \in(0, T), \text { and } \\
& \left(-|u|^{q-1} u(t)+|v|^{q-1} v(t)\right)_{+} \rightarrow 0 \text { in } L^{1}(\Omega) \text { as } t \searrow 0 .
\end{aligned}
$$

Theorem 3.6 (Comparison theorem [1]) Let $u$ and $v$ be a weak supersolution and subsolution to (3.1) in $\Omega_{T}$, respectively. If $u \geq v$ in the sense of (D3') on $\partial_{p} \Omega_{T}$, then it holds true that

$$
u \geq v \text { in } \Omega_{T} .
$$


Proof As before, for a small $\delta>0$, let us define the Lipschitz function $\phi_{\delta}$ by

$$
\phi_{\delta}(x):=\min \left\{1, \frac{x_{+}}{\delta}\right\}
$$

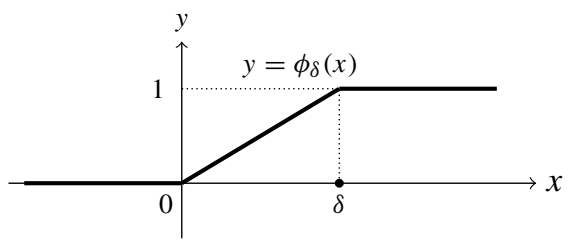

Note that $\phi_{\delta}(v-u) \in L^{\infty}\left(\Omega_{T}\right)$ and $L^{\infty}\left(0, T ; W_{0}^{1, p}(\Omega)\right)$. Let $0<t_{1}<t \leq T$ and $\sigma_{t_{1}, t}$ be the same time cut-off function as in the proof of Proposition 3.4. Choose a test function $\sigma_{t_{1}, t} \phi_{\delta}(v-u)$, which is admissible, to have

$$
\begin{aligned}
& \int_{\Omega_{t_{1}, t}} \partial_{t}\left(|u|^{q-1} u\right) \phi_{\delta}(v-u) \sigma_{t_{1}, t} \mathrm{~d} z+\int_{\Omega_{t_{1}, t}}|\nabla u|^{p-2} \nabla u \cdot \nabla\left(\phi_{\delta}(v-u)\right) \sigma_{t_{1}, t} \mathrm{~d} z \\
& \quad \geq c \int_{\Omega_{t_{1}, t}}|u|^{q-1} u \phi_{\delta}(v-u) \sigma_{t_{1}, t} \mathrm{~d} z
\end{aligned}
$$

and

$$
\begin{aligned}
& \int_{\Omega_{t_{1}, t}} \partial_{t}\left(|v|^{q-1} v\right) \phi_{\delta}(v-u) \sigma_{t_{1}, t} \mathrm{~d} z+\int_{\Omega_{t_{1}, t}}|\nabla v|^{p-2} \nabla v \cdot \nabla\left(\phi_{\delta}(v-u) \sigma_{t_{1}, t}\right) \mathrm{d} z \\
& \quad \leq c \int_{\Omega_{t_{1}, t}}|v|^{q-1} v \phi_{\delta}(v-u) \sigma_{t_{1}, t} \mathrm{~d} z
\end{aligned}
$$

Notice that

$$
\nabla \phi_{\delta}(v-u)= \begin{cases}\frac{1}{\delta}(\nabla v-\nabla u) & 0<v-u<\delta \\ 0 & \text { otherwise }\end{cases}
$$

and thus,

$$
\nabla \phi_{\delta}(v-u)=\frac{1}{\delta}(\nabla v-\nabla u) \chi_{\{0<v-u<\delta\}} .
$$

Subtract (3.14) from (3.15) in Lemma 2.4 to obtain

$$
\begin{aligned}
& \int_{\Omega_{t_{1}, t}} \partial_{t}\left(|v|^{q-1} v-|u|^{q-1} u\right) \phi_{\delta}(v-u) \sigma_{t_{1}, t} \mathrm{~d} z \\
& \leq-\int_{\Omega_{t_{1}, t}}\left(|\nabla v|^{p-2} \nabla v-|\nabla u|^{p-2} \nabla u\right) \cdot \frac{1}{\delta}(\nabla v-\nabla u) \chi_{\{0<v-u<\delta\}} \sigma_{t_{1}, t} \mathrm{~d} z \\
& \quad+c \int_{\Omega_{t_{1}, t}}\left(|v|^{q-1} v-|u|^{q-1} u\right) \phi_{\delta}(v-u) \sigma_{t_{1}, t} \mathrm{~d} z
\end{aligned}
$$


We find that the first term on the right hand side of (3.16) is bounded above as

$$
-\frac{c^{\prime}}{\delta} \int_{\Omega_{t_{1}, t}} \chi\{0<v-u<\delta\}|\nabla v-\nabla u|^{p} \sigma_{t_{1}, t} \mathrm{~d} z \leq 0
$$

for a positive constant $c^{\prime}$. Thus (3.16) and (3.17) lead to

$$
\begin{aligned}
& \int_{\Omega_{t_{1}, t}} \partial_{t}\left(|v|^{q-1} v-|u|^{q-1} u\right) \phi_{\delta}(v-u) \sigma_{t_{1}, t} \mathrm{~d} z \\
& \quad \leq c \int_{\Omega_{t_{1}, t}}\left(|v|^{q-1} v-|u|^{q-1} u\right) \phi_{\delta}(v-u) \sigma_{t_{1}, t} \mathrm{~d} z .
\end{aligned}
$$

Since $\partial_{t}\left(|u|^{q-1} u\right)$ and $\partial_{t}\left(|v|^{q-1} v\right)$ belong to $L^{2}\left(\Omega_{T}\right)$, by the Lebesgue's dominated convergence theorem, we can take the limit as $\delta \searrow 0$ in (3.18) and then, as $t_{1} \searrow 0$ to obtain

$$
\int_{\Omega}\left(|v|^{q-1} v(t)-|u|^{q-1} u(t)\right)_{+} \mathrm{d} x \leq c \int_{0}^{t} \int_{\Omega}\left(|v|^{q-1} v(\tau)-|u|^{q-1} u(\tau)\right)_{+} \mathrm{d} x \mathrm{~d} \tau
$$

where we used that $\phi_{\delta}(v-u) \rightarrow \chi_{\{v>u\}}$ as $\delta \searrow 0$ and that, from (2.10), $u \geq v$ is equivalent to $|u|^{q-1} u \geq|v|^{q-1} v$ and, by the initial trace condition,

$$
\lim _{t_{1} \rightarrow 0} \int_{\Omega}\left(|v|^{q-1} v\left(t_{1}\right)-|u|^{q-1} u\left(t_{1}\right)\right)_{+} \mathrm{d} x=0 .
$$

Thus Gronwall's lemma yields that

$$
\int_{\Omega}\left(|v|^{q-1} v(t)-|u|^{q-1} u(t)\right)_{+} \mathrm{d} x \leq 0
$$

and thus, $|v|^{q-1} v(t) \leq|u|^{q-1} u(t)$ in $\Omega, 0 \leq t \leq T$, which is equivalent to that $v(t) \leq u(t)$ in $\Omega, 0 \leq t \leq T$. Hence the proof is complete.

\subsection{Caccioppoli Type Estimates}

We present the Caccioppoli type estimates, which have a crucial role in De Giorgi's method (see Sect. 4). From Proposition 3.4 we find that if $u_{0} \geq 0$ in $\Omega$, a weak solution $u$ of (3.1) is nonnegative in $\Omega_{T}$. Thus we can consider (3.1) as

$$
\begin{cases}\partial_{t} u^{q}-\Delta_{p} u=c u^{q} & \text { in } \Omega_{T} \\ 0 \leq u \leq M & \text { on } \partial_{p} \Omega_{T}\end{cases}
$$

In what follows, we always assume that $u_{0} \geq 0$ in $\Omega$ and address (3.1') in place of (3.1). 
Let $K$ be a subset compactly contained in $\Omega$, and $0<t_{1}<t_{2} \leq T$. Here we use the notation $K_{t_{1}, t_{2}}=K \times\left(t_{1}, t_{2}\right)$. Let $\zeta$ be a smooth function such that $0 \leq \zeta \leq 1$ and $\zeta=0$ outside $K_{t_{1}, t_{2}}$. By use of $A^{+}(k, u)$ and $A^{-}(k, u)$, the local energy inequality can be derived.

Lemma 3.7 Let $k \geq 0$. Then following holds true:

(i) Let $u$ be a nonnegative weak supersolution to (3.1'). Then there exists a positive constant $C$ depending only on $p, n$ such that

$$
\begin{aligned}
& \underset{t_{1}<t<t_{2}}{\operatorname{ess} \sup _{K \times\{t\}}} A^{-}(k, u) \zeta^{p} \mathrm{~d} x+\int_{K_{t_{1}, t_{2}}}\left|\nabla(k-u)_{+} \zeta\right|^{p} \mathrm{~d} z \\
& \leq C \int_{K \times\left\{t_{1}\right\}} A^{-}(k, u) \zeta^{p} \mathrm{~d} x+C \int_{K_{t_{1}, t_{2}}}(k-u)_{+}^{p}|\nabla \zeta|^{p} \mathrm{~d} z \\
& \quad+C \int_{K_{t_{1}, t_{2}}} A^{-}(k, u) \zeta^{p-1}\left|\zeta_{t}\right| \mathrm{d} z
\end{aligned}
$$

(ii) Let $u$ be a nonnegative weak subsolution to (3.1'). Then there exists a positive constant $C$ depending only on $p, n$ such that

$$
\begin{aligned}
& \underset{t_{1}<t<t_{2}}{\operatorname{ess} \sup } \int_{K \times\{t\}} A^{+}(k, u) \zeta^{p} \mathrm{~d} x+\int_{K_{t_{1}, t_{2}}}\left|\nabla(u-k)_{+} \zeta\right|^{p} \mathrm{~d} z \\
& \quad \leq C \int_{K \times\left\{t_{1}\right\}} A^{+}(k, u) \zeta^{p} \mathrm{~d} x+C \int_{K_{t_{1}, t_{2}}}(u-k)_{+}^{p}|\nabla \zeta|^{p} \mathrm{~d} z \\
& \quad+C \int_{K_{t_{1}, t_{2}}} A^{+}(k, u) \zeta^{p-1}\left|\zeta_{t}\right| \mathrm{d} z+C \int_{K_{t_{1}, t_{2}}} c u^{q}(u-k)_{+} \zeta^{p} \mathrm{~d} z
\end{aligned}
$$

Proof We give the proof only for the case (i), because the case (ii) is treated by a similar argument. We note by (D1) in Definition 3.1 and the nonnegativity of $u$ in $\Omega_{T}$ that $\partial_{t} u^{q} \in L^{2}\left(\Omega_{T}\right)$. Choose a test function $\varphi$ as $-(k-u)_{+} \zeta^{p}$ in (D2) in Definition 3.1 to have

$$
\begin{aligned}
& -\int_{K_{t_{1}, t}} \frac{\partial u^{q}}{\partial t}(k-u)_{+} \zeta^{p} \mathrm{~d} z-\int_{K_{t_{1}, t}}|\nabla u|^{p-2} \nabla u \cdot \nabla\left((k-u)_{+} \zeta^{p}\right) \mathrm{d} z \\
& =-c \int_{K_{t_{1}, t}} u^{q}(k-u)_{+} \zeta^{p} \mathrm{~d} z \leq 0 .
\end{aligned}
$$

Using the formula (2.6), the first term on the left hand side of (3.21) is computed as

$$
\begin{aligned}
-\int_{K_{t_{1}, t}} \frac{\partial u^{q}}{\partial t}(k-u)_{+} \zeta^{p} \mathrm{~d} z & =\int_{K_{t_{1}, t}} \frac{\partial}{\partial t} A^{-}(k, u) \zeta^{p} \mathrm{~d} z \\
& =\left.\int_{K} A^{-}(k, u) \zeta^{p} \mathrm{~d} x\right|_{t_{1}} ^{t}-p \int_{K_{t_{1}, t}} A^{-}(k, u) \zeta^{p-1}\left|\zeta_{t}\right| \mathrm{d} z
\end{aligned}
$$


By use of Young's inequality, the second term on the left hand side of (3.21) is estimated from below by

$$
\frac{1}{2} \int_{K_{t_{1}, t}}\left|\nabla(k-u)_{+}\right|^{p} \zeta^{p} \mathrm{~d} z-C \int_{K_{t_{1}, t}}(k-u)_{+}^{p}|\nabla \zeta|^{p} \mathrm{~d} z .
$$

We gather (3.21), (3.22) and (3.23) to obtain, for any $t \in\left(t_{1}, t_{2}\right)$,

$$
\begin{aligned}
& \int_{K \times\{t\}} A^{-}(k, u) \zeta^{p} \mathrm{~d} x+\int_{K_{t_{1}, t}}\left|\nabla(k-u)_{+}\right|^{p} \zeta^{p} \mathrm{~d} z \\
& \leq C \int_{K \times\left\{t_{1}\right\}} A^{-}(k, u) \zeta^{p} \mathrm{~d} x+C \int_{K_{t_{1}, t}} A^{-}(k, u) \zeta^{p-1}\left|\zeta_{t}\right| \mathrm{d} z \\
& \quad+C \int_{K_{t_{1}, t}}(k-u)_{+}|\nabla \zeta|^{p} \mathrm{~d} z .
\end{aligned}
$$

Thus, we arrive at the conclusion.

The following so-called Caccioppoli type estimate follows from Lemma 3.7.

Proposition 3.8 (Caccioppoli type estimate) Let $k \geq 0$. Let $u$ be a nonnegative weak supersolution of (3.1). Then, there exists a positive constant $C$ depending only on $p, n$ such that

$$
\begin{aligned}
& \underset{t_{1}<t<t_{2}}{\operatorname{ess} \sup _{K \times\{t\}}} \int_{K}(k-u)_{+}^{q+1} \zeta^{p} \mathrm{~d} x+\int_{K_{t_{1}, t_{2}}}\left|\nabla(k-u)_{+} \zeta\right|^{p} \mathrm{~d} z \\
& \leq C \int_{K \times\left\{t_{1}\right\}} k^{q-1}(k-u)_{+}^{2} \zeta^{p} \mathrm{~d} x+C \int_{K_{t_{1}, t_{2}}}(k-u)_{+}^{p}|\nabla \zeta|^{p} \mathrm{~d} z \\
& \quad+C \int_{K_{t_{1}, t_{2}}} k^{q-1}(k-u)_{+}^{2}\left|\zeta_{t}\right| \mathrm{d} z .
\end{aligned}
$$

Proof We first estimate $A^{-}(k, u)=q \int_{u}^{k}(k-\eta)_{+} \eta^{q-1} \mathrm{~d} \eta$ defined in (2.4). The lower boundedness is obtained as follows:

Case $1(u \geq k / 2)$ : Since $\frac{k}{2} \leq u \leq \eta \leq k$ i.e., $\eta \geq k-\eta \geq 0$, it holds that

$$
A^{-}(k, u) \geq q \int_{u}^{k}(k-\eta)^{q} \mathrm{~d} \eta=\frac{q}{q+1}(k-u)^{q+1} .
$$

Case $2(u \leq k / 2)$ : $\quad$ Since $\frac{k}{2} \leq \eta \leq k$, i.e., $0 \leq k-\eta \leq \eta$, it holds that

$$
\begin{aligned}
A^{-}(k, u) & =q \int_{u}^{k / 2}(k-\eta)_{+} \eta^{q-1} \mathrm{~d} \eta+q \int_{k / 2}^{k}(k-\eta)_{+} \eta^{q-1} \mathrm{~d} \eta \\
& \geq q \int_{k / 2}^{k}(k-\eta)^{q} \mathrm{~d} \eta
\end{aligned}
$$




$$
\begin{aligned}
& =\frac{q}{q+1}\left(\frac{k}{2}\right)^{q+1} \\
& \geq \frac{q}{q+1} \frac{1}{2^{q+1}}(k-u)^{q+1},
\end{aligned}
$$

where, in the last line, we use $k>k-u \geq 0$ since $0 \leq u \leq k / 2$. Also, the upper boundedness follows from

$$
A^{-}(k, u)=q \int_{0}^{(k-u)_{+}}(k-\eta)^{q-1} \eta \mathrm{d} \eta \leq q k^{q-1} \int_{0}^{(k-u)_{+}} \eta \mathrm{d} \eta=q k^{q-1} \frac{(k-u)_{+}^{2}}{2} .
$$

Gathering Lemma 3.7, (3.26), (3.27) and (3.28), we arrive at the conclusion.

\section{Expansion of Positivity}

In this section, we will establish the expansion of positivity of a nonnegative solution to the doubly nonlinear equations of $p$-Sobolev flow type (3.1'). We make local estimates to show the expansion on space-time of positivity of a nonnegative weak (super)solution of (3.1').

\subsection{Expansion of Interior Positivity I}

In this subsection we will study expansion of local positivity of a weak solution of (3.1'). Following the argument in [7] (see also [6,15,26]), we proceed our local estimates.

Proposition 4.1 Let $u$ be a nonnegative weak supersolution of (3.1'). Let $B_{\rho}\left(x_{0}\right) \subset \Omega$ with center $x_{0} \in \Omega$ and radius $\rho>0$, and $t_{0} \in(0, T]$. Suppose that

$$
\left|B_{\rho}\left(x_{0}\right) \cap\left\{u\left(t_{0}\right) \geq L\right\}\right| \geq \alpha\left|B_{\rho}\right|
$$

holds for some $L>0$ and $\alpha \in(0,1]$. Then there exists positive numbers $\delta, \varepsilon \in(0,1)$ depending only on $p, n$ and $\alpha$ and independent of $L$ such that

$$
\left|B_{\rho}\left(x_{0}\right) \cap\{u(t) \geq \varepsilon L\}\right| \geq \frac{\alpha}{2}\left|B_{\rho}\right|
$$

for all $t \in\left[t_{0}, t_{0}+\delta L^{q+1-p} \rho^{p}\right]$. Here, if $t_{0}$ is very close to $T$, then $\delta$ is chosen so small that $\delta L^{q+1-p} \rho^{p}=T-t_{0}$.

Proof By a parallel translation invariance of the equation (3.1') we may assume $\left(x_{0}, t_{0}\right)=(0,0)$. For $k>0$ and $t>0$, let

$$
A_{k, \rho}(t):=B_{\rho} \cap\{u(t)<k\}
$$


Take a cutoff function $\zeta=\zeta(x)$ satisfying

$$
\xi \equiv 1 \quad \text { on } B_{(1-\sigma) \rho}, \quad|\nabla \zeta| \leq \frac{1}{\sigma \rho}
$$

where $\sigma$ is to be determined later. Applying the Caccioppoli type inequality (3.25) over $Q^{+}\left(\theta \rho^{p}, \rho\right):=B_{\rho} \times\left(0, \theta \rho^{p}\right)$ to the truncated function $(L-u)_{+}$and above $\zeta$, we obtain, for any $t \in\left(0, \theta \rho^{p}\right)$,

$$
\begin{aligned}
& \int_{B_{(1-\sigma) \rho}}(L-u(t))_{+}^{q+1} \mathrm{~d} x+C \int_{Q^{+}\left(\theta \rho^{p}, \rho\right)}\left|\nabla(L-u)_{+} \zeta\right|^{p} \mathrm{~d} z \\
& \leq \int_{B_{\rho}} L^{q-1}(L-u(0))_{+}^{2} \mathrm{~d} x+C \int_{Q^{+}(\theta \rho p, \rho)}(L-u)_{+}^{p}\left(\frac{1}{\sigma \rho}\right)^{p} \mathrm{~d} z \\
& \quad \leq L^{q+1}(1-\alpha)\left|B_{\rho}\right|+C \frac{\theta}{\sigma^{p}} L^{p}\left|B_{\rho}\right|,
\end{aligned}
$$

where we use the assumption (4.1) for $u(0)$, and thus, for any $t \in\left(0, \theta \rho^{p}\right)$,

$$
\int_{B_{(1-\sigma) \rho}}(L-u(t))_{+}^{q+1} \mathrm{~d} x \leq L^{q+1}\left\{(1-\alpha)+C \frac{\theta L^{p-(q+1)}}{\sigma^{p}}\right\}\left|B_{\rho}\right| .
$$

We will estimate the left hand side of (4.4). Firstly, we obtain that

$$
\begin{aligned}
\int_{B_{(1-\sigma) \rho}}(L-u(t))_{+}^{q+1} \mathrm{~d} x & \geq \int_{B_{(1-\sigma) \rho} \cap\{u(t)<\varepsilon L\}}(L-u)_{+}^{q+1} \mathrm{~d} x \\
& \geq L^{q+1}(1-\varepsilon)^{q+1}\left|A_{\varepsilon L,(1-\sigma) \rho}(t)\right| .
\end{aligned}
$$

Since $A_{\varepsilon L, \rho}(t) \backslash A_{\varepsilon L,(1-\sigma) \rho}(t) \subset B_{\rho} \backslash B_{(1-\sigma) \rho}$ and

$$
\begin{aligned}
\left|B_{\rho} \backslash B_{(1-\sigma) \rho}\right| & =\left|B_{1}(0)\right| \rho^{n}\left\{1-(1-\sigma)^{n}\right\} \\
& \leq\left|B_{1}(0)\right| \rho^{n}\{1-(1-n \sigma)\} \\
& \leq n \sigma\left|B_{\rho}\right|
\end{aligned}
$$

we have

$$
\begin{aligned}
\left|A_{\varepsilon L, \rho}(t)\right| & \leq\left|A_{\varepsilon L,(1-\sigma) \rho}(t)\right|+\left|A_{\varepsilon L, \rho}(t) \backslash A_{\varepsilon L,(1-\sigma) \rho}(t)\right| \\
& \leq\left|A_{\varepsilon L,(1-\sigma) \rho}(t)\right|+n \sigma\left|B_{\rho}\right| .
\end{aligned}
$$

From (4.5) and (4.6), we have

$$
\int_{B_{(1-\sigma) \rho}}(L-u(t))_{+}^{q+1} \mathrm{~d} x \geq L^{q+1}(1-\varepsilon)^{q+1}\left(\left|A_{\varepsilon L, \rho}(t)\right|-n \sigma\left|B_{\rho}\right|\right) .
$$


and thus, (4.4) and (4.7) yield that, for any $t \in\left(0, \theta \rho^{p}\right)$,

$$
\left|A_{\varepsilon L, \rho}(t)\right| \leq \frac{1}{(1-\varepsilon)^{q+1}}\left\{(1-\alpha)+C \frac{\theta L^{p-(q+1)}}{\sigma^{p}}+n \sigma\right\}\left|B_{\rho}\right| .
$$

Here we choose the parameters as

$$
\theta \leq \delta L^{q+1-p}, \quad \delta \leq \frac{\alpha^{p+1}}{2^{3 p+3} C n^{p}}, \quad \sigma=\frac{\alpha}{8 n}
$$

and $\varepsilon$ with

$$
\varepsilon \leq 1-\left\{\frac{1-\frac{3}{4} \alpha}{1-\frac{1}{2} \alpha}\right\}^{\frac{1}{q+1}}
$$

Then we find from (4.8)-(4.10) that, for any $t \in\left[0, \theta \rho^{p}\right]$,

$$
\left|A_{\varepsilon L, \rho}(t)\right| \leq \frac{1}{\frac{1-\frac{3}{4} \alpha}{1-\frac{1}{2} \alpha}} \cdot\left(1-\frac{3}{4} \alpha\right)\left|B_{\rho}\right|=\left(1-\frac{1}{2} \alpha\right)\left|B_{\rho}\right|
$$

that is, (4.2) is actually verified under (4.9), and thus the proof is complete.

Lemma 4.2 Let $u$ be a nonnegative weak supersolution of (3.1'). Let $Q_{4 \rho}\left(z_{0}\right):=$ $B_{4 \rho}\left(x_{0}\right) \times\left(t_{0}, t_{0}+\delta L^{q+1-p} \rho^{p}\right) \subset \Omega_{T}$, where $\delta$ is selected in Proposition 4.1. Then for any $v \in(0,1)$ there exists a positive number $\varepsilon_{v}$ depending only on $p, n, \alpha, \delta, v$ such that

$$
\left|Q_{4 \rho}\left(z_{0}\right) \cap\left\{u<\varepsilon_{v} L\right\}\right|<v\left|Q_{4 \rho}\right| .
$$

Proof We may assume $z_{0}=0$ as before. By Proposition 4.1, there exist positive numbers $\delta, \varepsilon \in(0,1)$ such that

$$
\left|B_{4 \rho} \cap\{u(t) \geq \varepsilon L\}\right| \geq \frac{\alpha}{2} \cdot 4^{-n}\left|B_{4 \rho}\right|
$$

for all $t \in\left[0, \delta L^{q+1-p} \rho^{p}\right]$.

Set $\theta=\delta L^{q+1-p}$ and let $\zeta=\zeta(x)$ be a piecewise smooth cutoff function satisfying

$$
\begin{aligned}
& 0 \leq \zeta \leq 1, \quad \zeta \equiv 0 \quad \text { oustside } B_{8 \rho} \\
& \zeta \equiv 1 \quad \text { on } \quad Q_{4 \rho}, \quad|\nabla \zeta| \leq(4 \rho)^{-1}
\end{aligned}
$$

From (4.13) and the Caccioppoli type inequality (3.25), applied for the truncated solution $\left(k_{j}-u\right)_{+}$over $Q_{4 \rho}$ with the level $k_{j}=\frac{1}{2^{j}} \varepsilon L(j=0,1, \ldots)$, we obtain 


$$
\begin{aligned}
\int_{Q_{4 \rho}}\left|\nabla\left(k_{j}-u\right)_{+}\right|^{p} \zeta^{p} \mathrm{~d} z \leq & \int_{B_{8 \rho} \times\{t=0\}} k_{j}^{q-1}\left(k_{j}-u\right)_{+}^{2} \zeta^{p} \mathrm{~d} x \\
& +C \int_{Q_{8 \rho}}\left(k_{j}-u\right)_{+}^{p}|\nabla \zeta|^{p} \mathrm{~d} z \\
\leq & C\left(k_{j}^{q+1}\left|B_{8 \rho}\right|+k_{j}^{p}\left|Q_{8 \rho}\right|(4 \rho)^{-p}\right) \\
\leq & C k_{j}^{p} L^{q+1-p}\left|B_{8 \rho}\right|\left(1+2^{-p} \delta\right) \\
\leq & C \frac{k_{j}^{p}}{\delta \rho^{p}}\left|Q_{8 \rho}\right|=C \frac{k_{j}^{p}}{\delta \rho^{p}}\left|Q_{4 \rho}\right| .
\end{aligned}
$$

Here we note that the constant $C$ depends only on $n, p$ and, in particular, is independent of $\rho, L$. On the other hand, applying De Giorgi's inequality (2.3) in Proposition 2.1 to $k=k_{j+1}$ and $l=k_{j}$, we have, for all $t, 0 \leq t \leq \delta L^{q+1-p} \rho^{p}=\theta \rho^{p}$,

$$
\begin{aligned}
& \left(k_{j}-k_{j+1}\right)\left|B_{4 \rho} \cap\left\{u(t)>k_{j}\right\}\right| \\
& \quad \leq C \frac{\rho^{n+1}}{\left|B_{4 \rho} \cap\left\{u(t)<k_{j+1}\right\}\right|} \int_{B_{4 \rho} \cap\left\{k_{j+1}<u(t)<k_{j}\right\}}|\nabla u(t)| \mathrm{d} x .
\end{aligned}
$$

Let $A_{j}(t):=B_{4 \rho} \cap\left\{u(t)<k_{j}\right\}$ and then, by (4.12), it holds that

$$
\left|B_{4 \rho} \backslash A_{j}(t)\right| \geq \frac{\alpha}{2} 4^{-n}\left|B_{4 \rho}\right|
$$

Combine (4.16) with (4.15) to have that

$$
\begin{aligned}
\frac{k_{j}}{2}\left|A_{j+1}(t)\right| & \leq \frac{\rho^{n+1}}{\left|B_{4 \rho} \backslash A_{j}(t)\right|} \int_{B_{4 \rho} \cap\left\{k_{j+1}<u(t)<k_{j}\right\}}|\nabla u(t)| \mathrm{d} x \\
& \leq \frac{C}{\alpha} \rho \int_{B_{4} \cap\left\{k_{j+1}<u(t)<k_{j}\right\}}|\nabla u(t)| \mathrm{d} x .
\end{aligned}
$$

Integrating above inequality (4.17) in $t \in\left(0, \theta \rho^{p}\right)$ yields

$$
\frac{k_{j}}{2}\left|A_{j+1}\right| \leq \frac{C}{\alpha} \rho \int_{Q_{4 \rho} \cap\left\{k_{j+1}<u<k_{j}\right\}}|\nabla u| \mathrm{d} z
$$

where we put $\left|A_{j}\right|:=\int_{0}^{\theta \rho^{p}}\left|A_{j}(t)\right| \mathrm{d} t=\left|Q_{4 \rho} \cap\left\{u(t)<k_{j}\right\}\right|$. By use of Hölder's inequality, (4.14) and (4.18), we have 


$$
\begin{aligned}
\frac{k_{j}}{2}\left|A_{j+1}\right| & \leq \frac{C}{\alpha} \rho\left[\int_{Q_{4 \rho}}\left|\nabla\left(k_{j}-u\right)_{+}\right|^{p} \mathrm{~d} z\right]^{\frac{1}{p}}\left|A_{j} \backslash A_{j+1}\right|^{\frac{p-1}{p}} \\
& \leq \frac{C}{\alpha} \rho \cdot \frac{k_{j}}{\delta^{\frac{1}{p}} \rho}\left|Q_{4 \rho}\right|^{\frac{1}{p}}\left(\left|A_{j}\right|-\left|A_{j+1}\right|\right)^{\frac{p-1}{p}} \\
& =\frac{C}{\alpha \delta^{\frac{1}{p}}} k_{j}\left|Q_{4 \rho}\right|^{\frac{1}{p}}\left(\left|A_{j}\right|-\left|A_{j+1}\right|\right)^{\frac{p-1}{p}}
\end{aligned}
$$

and thus,

$$
\left|A_{j+1}\right|^{\frac{p}{p-1}} \leq\left(\frac{C}{\alpha \delta^{\frac{1}{p}}}\right)^{\frac{p}{p-1}}\left|Q_{4 \rho}\right|^{\frac{1}{p-1}}\left(\left|A_{j}\right|-\left|A_{j+1}\right|\right) .
$$

Let $J \in \mathbb{N}$ be determined later. Summing (4.20) over $j=0,1, \ldots, J-1$, we obtain

$$
J\left|A_{J}\right|^{\frac{p}{p-1}} \leq\left(\frac{C}{\alpha \delta^{\frac{1}{p}}}\right)^{\frac{p}{p-1}}\left|Q_{4 \rho}\right|^{\frac{p}{p-1}}
$$

Indeed, by use of $\left|A_{0}\right| \geq\left|A_{j}\right| \geq\left|A_{J}\right|$ for $j \in\{0,1, \ldots, J\}$, we find that

$$
\sum_{j=0}^{J-1}\left|A_{j+1}\right|^{\frac{p}{p-1}} \geq J\left|A_{J}\right|^{\frac{p}{p-1}} \text { and } \sum_{j=0}^{J-1}\left(\left|A_{j}\right|-\left|A_{j+1}\right|\right) \leq\left|A_{0}\right| \leq\left|Q_{4 \rho}\right|
$$

Therefore, from (4.21), it follows that

$$
\left|A_{J}\right| \leq \frac{1}{J^{\frac{p-1}{p}}}\left(\frac{C}{\alpha \delta^{\frac{1}{p}}}\right)\left|Q_{4 \rho}\right|
$$

Thus, for any $v \in(0,1)$, we choose sufficiently large $J \in \mathbb{N}$ satisfying

$$
\frac{1}{J^{\frac{p-1}{p}}}\left(\frac{C}{\alpha \delta^{\frac{1}{p}}}\right) \leq v \Longleftrightarrow J \geq\left(\frac{C}{v \alpha \delta^{\frac{1}{p}}}\right)^{\frac{p}{p-1}}
$$

Here we note that $J$ depends only on $p, n, \alpha, \delta$ and $v$. We finally take $\varepsilon_{v}=\frac{\varepsilon}{2^{J}}$ and then (4.22) yields that

$$
\frac{\left|Q_{4 \rho} \cap\left\{u<\varepsilon_{v} L\right\}\right|}{\left|Q_{4 \rho}\right|}<v
$$

which is the very assertion. 
Remark 4.3 Look at the choice of $\delta$ in (4.9) and $\varepsilon$ in (4.10) in the proof of Proposition 4.1 , from which we can choose $\varepsilon$ such that

$$
\varepsilon=\left(\frac{\delta}{2^{I}}\right)^{\frac{1}{q+1-p}}
$$

for some large positive integer $I$. In the proof of Lemma 4.2 and the choice of $k_{j}$, we also choose $k_{j}$ as follows:

$$
k_{j}=\frac{\varepsilon L}{2^{\frac{j}{q+1-p}}} \text { for } j=0,1, \ldots, J
$$

Under such choice as above we note that $k_{J}=\left(\frac{\delta}{2^{I+J}}\right)^{\frac{1}{q+1-p}} L$ and obtain that

$$
\frac{\delta L^{q+1-p} \rho^{p}}{\left(k_{J}\right)^{q+1-p} \rho^{p}}=2^{J+I}
$$

which is a positive integer.

Following a similar argument to [7, p. 76], we next divide $Q_{4 \rho}\left(z_{0}\right)$ into finitely many subcylinders. For any $v \in(0,1)$, let $J$ be determined in (4.23). We divide $Q_{4 \rho}\left(z_{0}\right)$ along time direction into parabolic cylinders of number $s_{0}:=2^{I+J}$ with each timelength $k_{J}^{q+1-p} \rho^{p}$, and set

$$
Q^{(\ell)}:=B_{4 \rho}\left(x_{0}\right) \times\left(t_{0}+\ell k_{J}^{q+1-p} \rho^{p}, t_{0}+(\ell+1) k_{J}^{q+1-p} \rho^{p}\right)
$$

for $\ell=0,1, \ldots, s_{0}-1$.

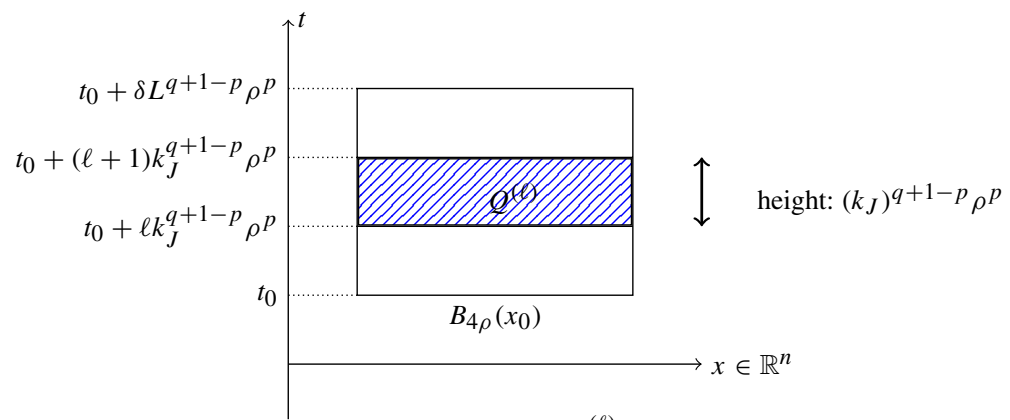

Figure: Image of $Q^{(\ell)}$

Then there exists a $Q^{(\ell)}$ which holds that

$$
\left|Q^{(\ell)} \cap\left\{u<k_{J}\right\}\right|<v\left|Q^{(\ell)}\right| \text {. }
$$

The following theorem enables us to have the positivity of a solution of (3.1') in a small interval. 
Theorem 4.4 (Expansion of local positivity) Let $u$ be a nonnegative weak supersolution of (3.1'). Let $Q_{4 \rho}\left(z_{0}\right)=B_{4 \rho}\left(x_{0}\right) \times\left(t_{0}, t_{0}+\delta L^{q+1-p} \rho^{p}\right) \subset \Omega_{T}$, where $\delta$ is chosen in Proposition 4.1. Suppose that (4.1). Under (4.26) there exists a positive number $\eta<1$ such that

$$
u \geq \eta L \quad \text { a.e. } \quad B_{2 \rho}\left(x_{0}\right) \times\left(t_{0}+\left(\ell+\frac{1}{2}\right) k_{J}^{q+1-p} \rho^{p}, t_{0}+(\ell+1) k_{J}^{q+1-p} \rho^{p}\right) .
$$

Proof Hereafter we fix the parameters $\rho, \ell$ and $k_{J}$. By translation we may assume to shift $\left(x_{0}, t_{0}+(\ell+1) k_{J}^{q+1-p} \rho^{p}\right)$ to the origin and thus, $Q^{(l)}$ is transformed to $B_{4 \rho}\left(x_{0}\right) \times\left(-k_{J}^{q+1-p} \rho^{p}, 0\right)$. For $m=0,1,2, \ldots$, let

$\tau_{m}=\frac{\rho^{p}}{2}\left(1+\frac{1}{2^{m}}\right), \quad \rho_{m}=\frac{\rho}{2}\left(1+\frac{1}{2^{m}}\right) ; \quad B_{m}:=B_{4 \rho_{m}}, \quad Q_{m}:=B_{m} \times\left(-\theta \tau_{m}, 0\right)$,

where $\theta:=k_{J}^{q+1-p}$, and also set

$$
\kappa_{m}:=\left(\frac{1}{2}+\frac{1}{2^{m+1}}\right) k_{J}
$$

It plainly holds true that

$$
\left\{\begin{array}{l}
\rho^{p}=\tau_{0} \geq \tau_{m} \searrow \tau_{\infty}=\rho^{p} / 2, \quad \rho=\rho_{0} \geq \rho_{m} \searrow \rho_{\infty}=\rho / 2, \\
Q_{0}=Q^{(\ell)} \supset Q_{m} \searrow Q_{\infty}=B_{2 \rho} \times\left(-\frac{1}{2} \theta \rho^{p}, 0\right), \\
k_{J}=\kappa_{0} \geq \kappa_{m} \searrow \kappa_{\infty}=k_{J} / 2 .
\end{array}\right.
$$

The cutoff function $\zeta$ is taken of the form $\zeta(x, t)=\zeta_{1}(x) \zeta_{2}(t)$, where $\zeta_{i}(i=1,2)$ are Lipschitz functions satisfying

$$
\zeta_{1}:=\left\{\begin{array}{lll}
1 & \text { in } & B_{m+1} \\
0 & \text { in } & \mathbb{R}^{n} \backslash B_{m}
\end{array}, \quad\left|\nabla \zeta_{1}\right| \leq \frac{1}{\rho_{m}-\rho_{m+1}}=\frac{2^{m+2}}{\rho}\right.
$$

and

$$
\zeta_{2}:=\left\{\begin{array}{ll}
0 & \text { for } t \leq-\theta \tau_{m} \\
1 & \text { for } t>-\theta \tau_{m+1}
\end{array}, \quad 0 \leq \zeta_{2, t} \leq \frac{1}{\theta\left(\tau_{m}-\tau_{m+1}\right)} \leq \frac{2^{p^{(m+2)}}}{\theta \rho^{p}}\right.
$$

Thus, applying the local energy inequality (3.25) over $B_{m}$ and $Q_{m}$ to the truncated solution $\left(\kappa_{m}-u\right)_{+}$and above $\zeta$, we obtain

$$
\begin{aligned}
& \underset{-\theta \tau_{m}<t<0}{\operatorname{ess} \sup } \int_{B_{m}}\left(\kappa_{m}-u(t)\right)_{+}^{q+1} \zeta^{p} \mathrm{~d} x+\int_{Q_{m}}\left|\nabla\left(\kappa_{m}-u\right)_{+}\right|^{p} \zeta^{p} \mathrm{~d} z \\
& \leq C \int_{Q_{m}}\left(\kappa_{m}-u\right)_{+}^{p}|\nabla \zeta|^{p} \mathrm{~d} z+C \int_{Q_{m}} \kappa_{m}^{q-1}\left(\kappa_{m}-u\right)_{+}^{2}\left|\zeta_{t}\right| \mathrm{d} z
\end{aligned}
$$




$$
\begin{aligned}
& \leq C\left(\frac{2^{m+2}}{\rho}\right)^{p} \kappa_{m}^{p} \int_{Q_{m}}\left(1+\frac{\kappa_{m}^{q+1-p}}{\theta}\right) \chi_{\left\{\left(\kappa_{m}-u\right)_{+}>0\right\}} \mathrm{d} z \\
& \leq C\left(\frac{2^{m+2}}{\rho}\right)^{p} \kappa_{m}^{p} \int_{Q_{m}} \chi_{\left\{\left(\kappa_{m}-u\right)_{+}>0\right\}} \mathrm{d} z,
\end{aligned}
$$

where we used that $\frac{\kappa_{m}^{q+1-p}}{\theta}=\left(\frac{\kappa_{m}}{k_{J}}\right)^{q+1-p} \leq 1$. Combining Proposition 2.2 with (4.28), we have

$$
\begin{aligned}
& \int_{Q_{m}}\left|\left(\kappa_{m}-u\right)_{+} \zeta\right|^{q+1} \mathrm{~d} z \\
& \quad=\int_{Q_{m}}\left|\left(\kappa_{m}-u\right)_{+} \zeta\right|^{p \frac{n+q+1}{n}} \mathrm{~d} z \\
& \leq C\left(\int_{Q_{m}}\left|\nabla\left[\left(\kappa_{m}-u\right)_{+} \zeta\right]\right|^{p} \mathrm{~d} z\right)\left(\operatorname{ess~sup~}_{-\theta \tau_{m}<t<0} \int_{B_{m}}\left|\left(\kappa_{m}-u(t)\right)_{+} \zeta\right|^{q+1} \mathrm{~d} z\right)^{\frac{p}{n}} \\
& \leq C\left(\frac{2^{m+2}}{\rho}\right)^{p\left(1+\frac{p}{n}\right)} k_{J}^{p\left(1+\frac{p}{n}\right)}\left(\int_{Q_{m}} \chi_{\left\{\left(\kappa_{m}-u\right)_{+}>0\right\}} \mathrm{d} z\right)^{1+\frac{p}{n}}
\end{aligned}
$$

where we note that $q+1=\frac{p(n+q+1)}{n}$ in the second line.

The left hand side of (4.29) is estimated from below as

$$
\begin{aligned}
\int_{Q_{m}}\left[\left(\kappa_{m}-u\right)_{+} \zeta\right]^{q+1} \mathrm{~d} z & \geq \int_{Q_{m}}\left[\left(\kappa_{m}-u\right)_{+} \zeta\right]^{q+1} \chi_{\left\{\left(\kappa_{m+1}-u\right)_{+}>0\right\}} \mathrm{d} z \\
& \geq\left|\kappa_{m}-\kappa_{m+1}\right|^{q+1} \int_{Q_{m+1}} \chi_{\left\{\left(\kappa_{m+1}-u\right)_{+}>0\right\}} \mathrm{d} z \\
& =\left(\frac{k_{J}}{2^{m+2}}\right)^{q+1} \int_{Q_{m+1}} \chi_{\left\{\left(\kappa_{m+1}-u\right)_{+}>0\right\}} \mathrm{d} z .
\end{aligned}
$$

Hence, by (4.29) and (4.30), we have

$$
\begin{aligned}
& \int_{Q_{m+1}} \chi_{\left\{\left(\kappa_{m+1}-u\right)_{+}>0\right\}} \mathrm{d} z \\
& \leq C \frac{\left[2^{p\left(1+\frac{p}{n}\right)+q+1}\right]^{m}}{\rho^{p\left(1+\frac{p}{n}\right)}} k_{J}^{p\left(1+\frac{p}{n}\right)-(q+1)}\left(\int_{Q_{m}} \chi_{\left\{\left(\kappa_{m}-u\right)_{+}>0\right\}} \mathrm{d} z\right)^{1+\frac{p}{n}},
\end{aligned}
$$

where we compute

$$
C\left(\frac{k_{J}}{2^{m+2}}\right)^{-(q+1)}\left(\frac{2^{m+1}}{\rho}\right)^{p\left(1+\frac{p}{n}\right)} k_{J}^{p\left(1+\frac{p}{n}\right)}=C \frac{\left[2^{p\left(1+\frac{p}{n}\right)+q+1}\right]^{m}}{\rho^{p\left(1+\frac{p}{n}\right)}} k_{J}^{p\left(1+\frac{p}{n}\right)-(q+1)} .
$$


Dividing the both side of (4.31) by $\left|Q_{m+1}\right|>0$, we have

$$
f_{Q_{m+1}} \chi_{\left\{\left(\kappa_{m+1}-u\right)_{+}>0\right\}} \mathrm{d} z \leq C\left[2^{p\left(1+\frac{p}{n}\right)+q+1}\right]^{m}\left(f_{Q_{m}} \chi_{\left\{\left(\kappa_{m}-u\right)_{+}>0\right\}} \mathrm{d} z\right)^{1+\frac{p}{n}}
$$

where

$$
\frac{\left|Q_{m}\right|^{1+\frac{p}{n}}}{\left|Q_{m+1}\right|} \leq C \rho^{p\left(1+\frac{p}{n}\right)}\left(k_{J}^{q+1-p}\right)^{\frac{p}{n}}
$$

and $p\left(1+\frac{p}{n}\right)-(q+1)+(q+1-p) \frac{p}{n}=0$ are used. Letting $Y_{m}:=f_{Q_{m}} \chi_{\left\{\left(\kappa_{m}-u\right)_{+}>0\right\}} \mathrm{d} z$, the above inequality (4.32) is rewritten as

$$
Y_{m} \leq C b^{m} Y_{m}^{1+\frac{p}{n}}, \quad m=0,1, \ldots
$$

where $b:=2^{p\left(1+\frac{p}{n}\right)+q+1}$. From Lemma 2.3, we find that if the initial value $Y_{0}$ satisfies

$$
Y_{0} \leq C^{-\left(\frac{n}{p}\right)} b^{-\left(\frac{n}{p}\right)^{2}}=: v_{0},
$$

then

$$
Y_{m} \rightarrow 0 \text { as } m \rightarrow \infty .
$$

Eq.(4.26) is equivalent to (4.33) by taking $v=v_{0}$, and then (4.34) leads to the conclusion (4.27) by putting $\eta=\frac{k_{J}}{2 L}<1$.

Remark 4.5 Theorem 4.4 asserts that the positivity propagates after the lapse of some time. If a solution is positive at some time $t_{0}$, its positivity expands in space-time without "waiting time", which is in the next corollary.

Corollary 4.6 Let $u$ be a nonnegative weak supersolution of (3.1'). Assume that $u\left(t_{0}\right)>$ 0 in $B_{4 \rho}\left(x_{0}\right) \subset \Omega$. Then there exist positive numbers $\eta_{0}$ and $\tau_{0}$ such that

$$
u \geq \eta_{0} \quad \text { a.e. in } B_{2 \rho}\left(x_{0}\right) \times\left(t_{0}, t_{0}+\tau_{0}\right) .
$$

Proof Suppose that $\left(x_{0}, t_{0}\right)$ be the origin, as before. Let $L:=\inf _{B_{4} \rho} u(0)>0$. Since $\left|B_{\rho} \cap\{u(0) \geq L\}\right|=\left|B_{\rho}\right|$, by Proposition 4.1 , there exist positive numbers $\delta, \varepsilon$ depending only on $n, p$ and independent of $L$ such that

$$
\left|\{u(t) \geq \varepsilon L\} \cap B_{\rho}\right| \geq \frac{1}{2}\left|B_{\rho}\right|
$$

for all $t \in\left[0, \delta L^{q+1-p} \rho^{p}\right]$. Let $Q_{4 \rho}^{\theta}\left(z_{0}\right)=B_{4 \rho}\left(x_{0}\right) \times\left(0, \theta \rho^{p}\right) \in \Omega_{T}$, where $0<$ $\theta \leq \delta L^{q+1-p}$. By Lemma 4.2 with some minor change, for any $v \in(0,1)$ there exists a positive number $\varepsilon_{v}$ depending only on $p, n, \delta$ and $v$ such that

$$
\left|Q_{4 \rho}^{\theta}\left(z_{0}\right) \cap\left\{u<\varepsilon_{v} L\right\}\right|<v\left|Q_{4 \rho}^{\theta}\right| .
$$


Here we notice that in the proof of Proposition 4.1 and Lemma 4.2, we do not need to use the cutoff on time. In the following we modify the proof of Theorem 4.4 to that without any cutoff on time.

By translation, we may assume $x_{0}=0$ as before. For $m=0,1, \ldots$, we put

$$
\rho_{m}:=\left(2+\frac{1}{2^{m-1}}\right) \rho, \quad Q_{m}:=B_{\rho_{m}} \times\left(0, \theta \rho^{p}\right), \quad \theta \leq \delta L^{q+1-p}
$$

and also set

$$
\kappa_{m}:=\left(\frac{1}{2}+\frac{1}{2^{m+1}}\right) k_{J}
$$

where $k_{J}=\frac{\varepsilon L}{2^{J}}$ and $J$ is to be determined in (4.23).

Clearly it holds true that

$$
\left\{\begin{array}{l}
4 \rho=\rho_{0} \geq \rho_{m} \searrow \rho_{\infty}=2 \rho, \quad Q_{0} \supset Q_{m} \searrow Q_{\infty}=B_{2 \rho} \times\left(0, \theta \rho^{p}\right) \\
k_{J}=\kappa_{0} \geq \kappa_{m} \searrow \kappa_{\infty}=k_{J} / 2
\end{array}\right.
$$

A cutoff function $\zeta$ is chosen of the form $\zeta(x, t)=\zeta_{1}(x)$, where

$$
\zeta_{1}=\left\{\begin{array}{ll}
1 & x \in B_{\rho_{m+1}} \\
0 & x \in \mathbb{R}^{n} \backslash B_{\rho_{m}}
\end{array} \quad, \quad\left|\nabla \zeta_{1}(x)\right| \leq \frac{1}{\rho_{m}-\rho_{m+1}}=\frac{2^{m+1}}{\rho^{m}} .\right.
$$

From the Caccioppoli type inequality (3.25), applied for the truncated solution $\left(\kappa_{m}-u\right)_{+}$in $Q_{m}$ again, we obtain that

$$
\begin{aligned}
& \underset{0<t<\theta \rho}{\operatorname{ess} \sup _{p}} \int_{B_{m}}\left(\kappa_{m}-u(t)\right)_{+}^{q+1} \zeta^{p} \mathrm{~d} x+\int_{Q_{m}}\left|\nabla\left(\kappa_{m}-u\right)_{+}\right|^{p} \zeta^{p} \mathrm{~d} z \\
& \quad \leq \int_{B_{m}} \kappa_{m}^{q-1}\left(\kappa_{m}-u(0)\right)_{+}^{2} \zeta^{p} \mathrm{~d} x+C \int_{Q_{m}}\left(\kappa_{m}-u\right)_{+}^{p}|\nabla \zeta|^{p} \mathrm{~d} z \\
& \quad \leq C\left(\frac{\rho}{2^{m+1}}\right)^{p} \kappa_{m}^{p} \int_{Q_{m}} \chi_{\left\{\left(\kappa_{m}-u\right)_{+}>0\right\}} \mathrm{d} z,
\end{aligned}
$$

where, in the second line, we used $\left(\kappa_{m}-u(0)\right)_{+}=0$ in $B_{m}$. By the very same argument as in the proof of Theorem 4.4 , we have that

$$
f_{Q_{m+1}} \chi_{\left\{\left(\kappa_{m+1}-u\right)_{+}>0\right\}} \mathrm{d} z \leq C\left[2^{p\left(1+\frac{p}{n}\right)+q+1}\right]^{m}\left(f_{Q_{m}} \chi_{\left\{\left(\kappa_{m}-u\right)_{+}>0\right\}} \mathrm{d} z\right)^{1+\frac{p}{n}} .
$$

Letting $Y_{m}:=\int_{Q_{m}} \chi_{\left\{\left(\kappa_{m}-u\right)_{+}>0\right\}} \mathrm{d} z$, the above inequality (4.38) is rewritten as:

$$
Y_{m+1} \leq C b^{m} Y_{m}^{1+\frac{p}{n}}, \quad m=0,1, \ldots,
$$


where $b:=2^{p\left(1+\frac{p}{n}\right)+q+1}>1$. By Lemma 2.3 on fast geometric convergence if

$$
Y_{0} \leq C^{-\frac{n}{p}} b^{-\left(\frac{n}{p}\right)^{2}}=: v_{0}
$$

then

$$
Y_{m} \rightarrow 0 \text { as } m \rightarrow \infty .
$$

Eq. (4.39) is equivalent to (4.36) by taking $v=v_{0}$, and then (4.40) yields that

$$
u \geq \eta L \quad \text { a.e. in } B_{2 \rho} \times\left(0, \theta \rho^{p}\right)
$$

where $\eta=\frac{1}{2} \frac{\varepsilon}{2^{J}}$ and $\theta \leq \delta L^{q+1-p}$. Thus, letting $\eta_{0}:=\eta L$ and $\tau_{0}:=\theta \rho^{p}$, we reach the conclusion.

\subsection{Expansion of Interior Positivity II}

We continue to study the expansion of positivity of a nonnegative solution. Let $\Omega^{\prime}$ be a subdomain contained compactly in $\Omega$. Using Theorem 4.4 and a method of chain of finitely many balls as used in Harnack's inequality for harmonic functions, which is so-called Harnack chain (see [8, Theorem 11, pp. 32-33] and [4,17] in the p-parabolic setting), we have the following theorem. Here we use the special choice of parameters, as explained before Theorem 4.4.

Theorem 4.7 Let $u$ be a nonnegative weak supersolution of (3.1'). Let $\Omega^{\prime}$ be a subdomain contained compactly in $\Omega$. Let $0<\rho \leq \operatorname{dist}\left(\Omega^{\prime}, \partial \Omega\right) / 4$, and $t_{0} \in(0, T]$. Suppose that

$$
\left|\Omega^{\prime} \cap\left\{u\left(t_{0}\right) \geq L\right\}\right| \geq \alpha\left|\Omega^{\prime}\right|
$$

holds for some $L>0$ and $\alpha \in(0,1]$. Then there exist positive integer $N=N\left(\Omega^{\prime}\right)$, positive real number families $\left\{\delta_{m}\right\}_{m=0}^{N},\left\{\eta_{m}\right\}_{m=1}^{N+1} \subset(0,1),\left\{J_{m}\right\}_{m=0}^{N},\left\{I_{m}\right\}_{m=0}^{N} \subset \mathbb{N}$ depending on $p, n, \alpha$ and independent of $L$, and a time $t_{N}>t_{0}$ such that

$$
u \geq \eta_{N+1} L
$$

almost everywhere in

$$
\Omega^{\prime} \times\left(t_{N}+\left(k+\frac{1}{2}\right) \frac{\delta_{N}\left(\eta_{N} L\right)^{q+1-p}}{2^{J_{N}+I_{N}}} \rho^{p}, t_{N}+(k+1) \frac{\delta_{N}\left(\eta_{N} L\right)^{q+1-p}}{2^{J_{N}+I_{N}}} \rho^{p}\right)
$$

for some $k \in\left\{0,1, \ldots, 2^{J_{N}+I_{N}}-1\right\}$, where $t_{N}$ is written as

$$
t_{N}=t_{0}+\sum_{m=1}^{N}\left(\ell+\frac{3}{4}\right) \frac{\delta_{m-1}\left(\eta_{m-1} L\right)^{q+1-p}}{2^{J_{m-1}+I_{m-1}}} \rho^{p}
$$

for some $\ell \in\left\{0,1, \ldots, 2^{J_{m-1}+I_{m-1}-1}\right\}$. 
Proof We will prove the assertion in five steps.

Step 1: Since $\overline{\Omega^{\prime}}$ is compact, it is covered by finitely many balls $\left\{B_{\rho}\left(x_{j}\right)\right\}_{j=1}^{N}\left(x_{j} \in\right.$ $\left.\Omega^{\prime}, \overline{j=1}, 2, \ldots, N\right)$, where $N=N\left(\Omega^{\prime}\right)$, such that

$$
\overline{\Omega^{\prime}}=\bigcup_{j=1}^{N} \overline{B_{\rho}}\left(x_{j}\right), \quad \rho<\left|x_{i}-x_{i+1}\right|<2 \rho, \quad B_{4 \rho}\left(x_{i}\right) \subset \Omega, \text { for all } 1 \leq i \leq N,
$$

where we put $x_{N+1}=x_{1}$. For brevity we denote $B_{\rho}\left(x_{j}\right)$ by $B_{j}$ for each $j=1,2, \ldots, N$ and let $\sigma B_{j}:=B_{\sigma \rho}\left(x_{j}\right)$ for $\sigma=2$ and 4 .

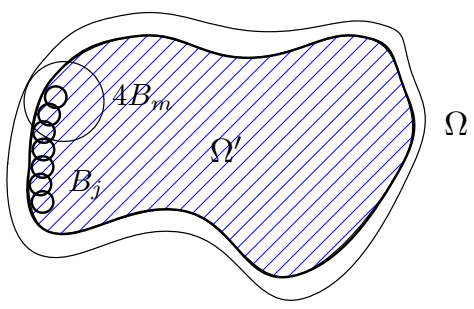

Figure: Harnack's chain argument

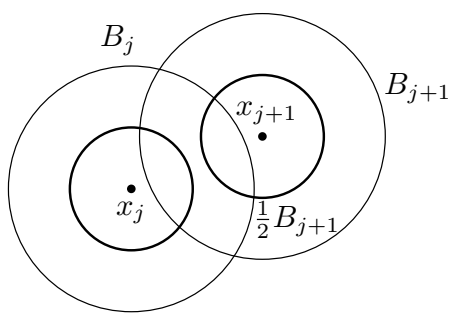

Figure: Relations of two balls

By (4.41), there exists at least one $B_{j}=B_{\rho}\left(x_{j}\right)$, denoted by $x_{1}=x_{j}$ and $B_{1}=B_{j}$, such that

$$
\left|B_{1} \cap\left\{u\left(t_{0}\right) \geq L\right\}\right| \geq \frac{\alpha}{2^{n}}\left|B_{1}\right|,
$$

Thus, by Proposition 4.1, there exist positive numbers $\delta_{0}, \varepsilon_{0} \in(0,1)$ depending only on $p, n$ and $\alpha_{0}=\alpha$ and independent of $L$ such that

$$
\left|B_{1} \cap\left\{u(t) \geq \varepsilon_{0} L\right\}\right| \geq \frac{\alpha_{0}}{2^{n+1}}\left|B_{1}\right| .
$$

for all $t \in\left[t_{0}, t_{0}+\delta_{0} L^{q+1-p} \rho^{p}\right]$. Let $Q_{4 \rho}\left(z_{1}\right):=4 B_{1} \times\left(t_{0}, t_{0}+\delta_{0} L^{q+1-p} \rho^{p}\right) \subset \Omega_{T}^{\prime}$. By Lemma 4.2, for any $\nu_{0} \in(0,1)$, there exists a positive number $\varepsilon_{v_{0}}$ depending only on $p, n, \alpha_{0}, \delta, v_{0}, \varepsilon_{0}$ such that

$$
\left|Q_{4 \rho}\left(z_{1}\right) \cap\left\{u<\varepsilon_{\nu_{0}} L\right\}\right|<v_{0}\left|Q_{4 \rho}\left(z_{1}\right)\right| .
$$

Here $\varepsilon_{v_{0}}:=\varepsilon_{0} / 2^{J_{0}}$, where $J_{0}$ is determined in (4.23) replaced $v$ by $v_{0}$. In particular, as noted in (4.24), we choose $\varepsilon_{0}$ as $\varepsilon_{0}=\left(\frac{\delta_{0}}{2^{I_{0}}}\right)^{\frac{1}{q+1-p}}$, where $I_{0}$ is sufficiently large positive integer. Following the same argument as before, we next divide $Q_{4 \rho}\left(z_{1}\right)$ along time direction into finitely many subcylinders of number $s_{0}:=2^{J_{0}+I_{0}}$, with each time-length

$$
k_{J_{0}}^{q+1-p} \rho^{p}=\frac{\delta_{0} L^{q+1-p}}{2^{J_{0}+I_{0}}} \rho^{p}
$$


via (4.25) and put

$$
Q^{(\ell)}\left(z_{1}\right):=4 B_{1} \times\left(t_{0}+\ell \frac{\delta_{0} L^{q+1-p}}{2^{J_{0}+I_{0}}} \rho^{p}, t_{0}+(\ell+1) \frac{\delta_{0} L^{q+1-p}}{2^{J_{0}+I_{0}}} \rho^{p}\right)
$$

for $\ell=0,1, \ldots, s_{0}-1$. Thus, by (4.42), there exists a $Q^{(\ell)}\left(z_{1}\right)$ such that

$$
\left|Q^{(\ell)}\left(z_{1}\right) \cap\left\{u<\varepsilon_{v_{0}} L\right\}\right|<v_{0}\left|Q^{(\ell)}\left(z_{1}\right)\right|
$$

Therefore it follows from Theorem 4.4 that

$u \geq \eta_{1} L$ a.e. in $2 B_{1} \times\left(t_{0}+\left(\ell+\frac{1}{2}\right) \frac{\delta_{0} L^{q+1-p}}{2^{J_{0}+I_{0}}} \rho^{p}, t_{0}+(\ell+1) \frac{\delta_{0} L^{q+1-p}}{2^{J_{0}+I_{0}}} \rho^{p}\right)$,

where $\eta_{1}:=\frac{1}{2 L} k_{J_{0}}$.

Step 2: By $\rho<\left|x_{1}-x_{2}\right|<2 \rho$,

$$
D_{1}:=B_{1} \cap B_{2} \neq \varnothing
$$

holds.

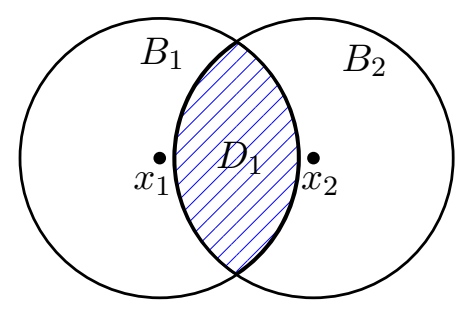

Figure: Intersection of two balls

Via (4.43), we have

$$
u \geq \eta_{1} L \text { a.e. } D_{1} \times \mathcal{I}_{0}^{(\ell)},
$$

where let $\mathcal{I}_{0}^{(\ell)}:=\left(t_{0}+\left(\ell+\frac{1}{2}\right) \frac{\delta_{0} L^{q+1-p}}{2^{J_{0}+I_{0}}} \rho^{p}, t_{0}+(\ell+1) \frac{\delta_{0} L^{q+1-p}}{2^{J_{0}+I_{0}}} \rho^{p}\right)$. Let $t_{1}$ be a middle point in $\mathcal{I}_{0}^{(\ell)}$ and, by (4.44), we observe that

$$
\left|D_{1} \cap\left\{u\left(t_{1}\right) \geq \eta_{1} L\right\}\right|=\left|D_{1}\right|,
$$

which is, setting $\alpha_{1}:=\frac{\left|D_{1}\right|}{\left|B_{2}\right|} \in(0,1)$,

$$
\left|B_{2} \cap\left\{u\left(t_{1}\right) \geq \eta_{1} L\right\}\right| \geq \alpha_{1}\left|B_{2}\right| .
$$


By the very same argument as Step 1 , there exist positive numbers $\delta_{1} \in(0,1), I_{1}, J_{1} \in$ $\mathbb{N}$ depending only on $p, n$ and $\alpha_{1}$ and independent of $L$, and $\nu_{1} \in(0,1)$ such that

$$
\begin{aligned}
& u \geq \eta_{2} L \text { a.e. } \\
& \text { in } B_{2} \times\left(t_{1}+\left(k+\frac{1}{2}\right) \frac{\delta_{1}\left(\eta_{1} L\right)^{q+1-p}}{2^{J_{1}+I_{1}}} \rho^{p}, t_{1}+(k+1) \frac{\delta_{1}\left(\eta_{1} L\right)^{q+1-p}}{2^{J_{1}+I_{1}}} \rho^{p}\right),
\end{aligned}
$$

for some

$$
k \in\left\{0,1, \ldots, 2^{I_{1}+J_{1}}-1\right\},
$$

where $\eta_{2}:=\frac{1}{2 L} k_{J_{1}}$ and $J_{1}$ is chosen that

$$
J_{1} \geq \max \left\{\left(\frac{C}{\nu_{1} \alpha_{1} \delta_{1}^{\frac{1}{p}}}\right)^{\frac{p}{p-1}}, J_{0}\right\} .
$$

Step 3: We will proceed by induction on $m$. Assume that for some $m \in$ $\{1, \overline{2 \ldots}, N\}$

$$
u \geq \eta_{m} L \text { a.e. in } B_{m} \times \mathcal{I}_{m-1}^{(\ell)} .
$$

Here we let

$$
\begin{aligned}
\mathcal{I}_{m-1}^{(\ell)}:= & \left(t_{m-1}+\left(\ell+\frac{1}{2}\right) \frac{\delta_{m-1}\left(\eta_{m-1} L\right)^{q+1-p}}{2^{J_{m-1}+I_{m-1}}} \rho^{p}, t_{m-1}\right. \\
& \left.+(\ell+1) \frac{\delta_{m-1}\left(\eta_{m-1} L\right)^{q+1-p}}{2^{J_{m-1}+I_{m-1}}} \rho^{p}\right)
\end{aligned}
$$

where $\delta_{m-1}, \eta_{m-1} \in(0,1)$ and $J_{m-1}, I_{m-1} \in \mathbb{N}$ are determined inductively, and $\ell \in\left\{0,1, \ldots, 2^{I_{m-1}+J_{m-1}}-1\right\}$. By $\rho<\left|x_{m}-x_{m+1}\right|<2 \rho$ again,

$$
D_{m}:=B_{m} \cap B_{m+1} \neq \varnothing
$$

and thus, (4.46) yields that

$$
u \geq \eta_{m} L \text { a.e. in } D_{m} \times \mathcal{I}_{m-1}^{(\ell)} .
$$

Letting $t_{m}$ be a middle point of $\mathcal{I}_{m-1}^{(\ell)}$ again and, by (4.47), we obtain that

$$
\left|B_{m+1} \cap\left\{u\left(t_{m}\right) \geq \eta_{m} L\right\}\right| \geq \alpha_{m}\left|B_{m+1}\right|,
$$


where we let $\alpha_{m}:=\frac{\left|D_{m}\right|}{\left|B_{m+1}\right|} \in(0,1)$. Again, using the very same argument as in Step 1 and 2 , there exist positive numbers $\delta_{m}, \eta_{m} \in(0,1), J_{m}, I_{m} \in \mathbb{N}$ depending only on $p, n$ and $\alpha_{m}$ and independent of $L$ and some $v_{m} \in(0,1)$ such that

$$
u \geq \eta_{m+1} L \text { a.e. in } B_{m+1} \times \mathcal{I}_{m}^{(k)},
$$

where

$$
\mathcal{I}_{m}^{(k)}:=\left(t_{m}+\left(k+\frac{1}{2}\right) \frac{\delta_{m}\left(\eta_{m} L\right)^{q+1-p}}{2^{J_{m}+I_{m}}} \rho^{p}, t_{m}+(k+1) \frac{\delta_{m}\left(\eta_{m} L\right)^{q+1-p}}{2^{J_{m}+I_{m}}} \rho^{p}\right)
$$

for some

$$
k \in\left\{0,1, \ldots, 2^{I_{m}+J_{m}}-1\right\}
$$

where

$$
\eta_{m+1}=\frac{1}{2 L} k_{J_{m}}=\left(\frac{\delta_{m}}{2^{I_{m}+J_{m}}}\right)^{\frac{1}{q+1-p}} \eta_{m}
$$

and $J_{m}$ is chosen that

$$
J_{m} \geq \max \left\{\left(\frac{C}{v_{m} \alpha_{m} \delta_{m}^{\frac{1}{p}}}\right)^{\frac{p}{p-1}}, J_{m-1}\right\} .
$$

Step 4: We next claim the following:

For any $k \in\left\{0,1, \ldots, 2^{I_{m}+J_{m}}-1\right\}$ and $\ell \in\left\{0,1, \ldots, 2^{I_{m-1}+J_{m-1}}-1\right\}$,

$$
\mathcal{I}_{m-1}^{(\ell)} \supset \mathcal{I}_{m}^{(k)}
$$

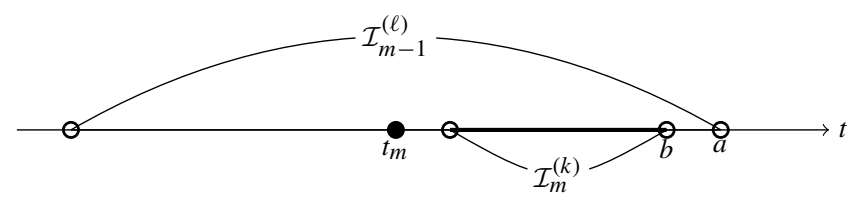

Figure: Relations of $\mathcal{I}_{m-1}^{(\ell)}$ and $\mathcal{I}_{m}^{(\ell)}$

Since this (4.50) is equivalent to

$$
a=t_{m-1}+(\ell+1) \frac{\delta_{m-1}\left(\eta_{m-1} L\right)^{q+1-p}}{2^{J_{m-1}+I_{m-1}}} \rho^{p}>t_{m}+(k+1) \frac{\delta_{m}\left(\eta_{m} L\right)^{q+1-p}}{2^{J_{m}+I_{m}}} \rho^{p}=b,
$$

we will verify (4.51). Using $\eta_{m}=\frac{1}{2 L} k_{J_{m-1}}=\left(\frac{\delta_{m-1}}{2^{I m-1+J_{m-1}}}\right)^{\frac{1}{q+1-p}} \eta_{m-1}$ via (4.49) and

$$
t_{m}=t_{m-1}+\left(\ell+\frac{3}{4}\right) \frac{\delta_{m-1}\left(\eta_{m-1} L\right)^{q+1-p}}{2^{J_{m-1}+I_{m-1}}} \rho^{p}: \text { middle point of } \mathcal{I}_{m-1}^{(\ell)},
$$


we find that $a-b$ is estimated as follows:

$$
\begin{aligned}
a-b & =\left[\frac{1}{4}-(k+1) \frac{\delta_{m}}{2^{J_{m}+I_{m}}}\right] \frac{\delta_{m-1}\left(\eta_{m-1} L\right)^{q+1-p}}{2^{J_{m-1}+I_{m-1}}} \rho^{p} \\
& >\left[\frac{1}{4}-2^{J_{m}+I_{m}} \cdot \frac{\delta_{m}}{2^{J_{m}+I_{m}}}\right] \frac{\delta_{m-1}\left(\eta_{m-1} L\right)^{q+1-p}}{2^{J_{m-1}+I_{m-1}}} \rho^{p} \\
& >\left[\frac{1}{4}-\delta_{m}\right] \frac{\delta_{m-1}\left(\eta_{m-1} L\right)^{q+1-p}}{2^{J_{m-1}+I_{m-1}}} \rho^{p} \\
& >0
\end{aligned}
$$

since $\delta_{m}=\frac{\alpha_{m}^{p+1}}{2^{3 p+3} \mathrm{Cn}^{p}}<\frac{1}{4}$ by (4.9). Thus, (4.50) is actually valid.

Step 5: By Step 3, we have, for all $m=1,2, \ldots, N$,

$$
u \geq \eta_{m+1} L \text { a.e. in } B_{m+1} \times \mathcal{I}_{m}^{(k)},
$$

where we put $B_{N+1}:=B_{1}$. Since $\left\{\eta_{m}\right\}_{m=1}^{N+1}$ is decreasing this inequality plainly yields that, for all $m=1,2, \ldots, N$,

$$
u \geq \eta_{N+1} L \text { a.e. in } B_{m+1} \times \mathcal{I}_{m}^{(k)} .
$$

By (4.50) in Step 4, we furthermore find that

$$
\mathcal{I}_{1}^{(\ell)} \supset \cdots \supset \mathcal{I}_{N}^{(k)}
$$

where $\ell \in\left\{0,1, \ldots, 2^{J_{0}+I_{0}}-1\right\}$ and $k \in\left\{0,1, \ldots, 2^{J_{N}+I_{N}}-1\right\}$. From (4.52) and (4.53) it follows that

$$
u \geq \eta_{N+1} L \text { a.e. in } \Omega^{\prime} \times \mathcal{I}_{N}^{(k)}
$$

and thus, we arrive at the conclusion.

As mentioned in Corollary 4.6, if a solution of (3.1') is positive in $\Omega$ at some time $t_{0}$, its positivity expands in space-time without "waiting time".

Corollary 4.8 Let $u$ be a nonnegative weak supersolution of (3.1'). Let $\Omega^{\prime}$ be a subdomain contained compactly in $\Omega$. Suppose that $u\left(t_{0}\right)>0$ in $\Omega$ for some $t_{0} \in[0, T)$. Then there exist positive numbers $\eta_{0}$ and $\tau_{0}$ such that

$$
u \geq \eta_{0} \text { a.e. in } \Omega^{\prime} \times\left(t_{0}, t_{0}+\tau_{0}\right) .
$$

Proof Since $\overline{\Omega^{\prime}}$ is compact, it is covered by finitely many balls $\left\{B_{\rho}\left(x_{j}\right)\right\}_{j=1}^{N}\left(x_{j} \in\right.$ $\left.\Omega^{\prime}, j=1,2, \ldots, N\right)$, where $N=N\left(\Omega^{\prime}\right)$ is a positive integer such that

$$
\overline{\Omega^{\prime}}=\bigcup_{j=1}^{N} \overline{B_{\rho}}\left(x_{j}\right), \quad \rho<\left|x_{i}-x_{i+1}\right|<2 \rho, \quad B_{2 \rho}\left(x_{i}\right) \subset \Omega, \text { for all } 1 \leq i \leq N,
$$


where we put $x_{N+1}=x_{1}$. For brevity we denote $B_{\rho}\left(x_{j}\right)$ by $B_{j}$ for each $j=$ $1,2, \ldots, N$ and let $2 B_{j}:=B_{2 \rho}\left(x_{j}\right)$. By assumption, $u>0$ in each ball $2 B_{j}$, $j=1, \ldots, N$. Corollary 4.6 yields that there exist positive numbers $\eta_{1}$ and $\tau_{1}$ such that

$$
u \geq \eta_{1} \text { a.e. in } B_{1} \times\left(t_{0}, t_{0}+\tau_{1}\right) .
$$

Similarly as above, it follows that there exist positive numbers $\tau_{2}<\tau_{1}, \eta_{2}<\eta_{1}$ such that

$$
u \geq \eta_{2} \text { a.e. in } B_{2} \times\left(t_{0}, t_{0}+\tau_{2}\right) .
$$

Iterative this argument finitely, there exist $\eta_{1}>\eta_{2}>\cdots>\eta_{N}$ and $\tau_{1}>\tau_{2}>\cdots>$ $\tau_{N}$ such that

$$
u \geq \eta_{N} \quad \text { a.e. in } B_{j} \times\left(t_{0}, t_{0}+\tau_{N}\right) .
$$

for all $j=1, \ldots, N$. Thus, letting $\eta_{0}:=\eta_{N}$ and $\tau_{0}:=\tau_{N}$, we complete the proof.

\subsection{Positivity Near the Boundary}

We next study the positivity of the solution to the doubly nonlinear equations of $p$ Sobolev flow type (3.1') near the boundary. In what follows, assume that the bounded domain $\Omega$ satisfies the interior ball condition, that is, for every boundary point $\xi \in \partial \Omega$, there exist a point $x_{0} \in \Omega$ and some $\rho>0$ such that

$$
\overline{B_{\rho}\left(x_{0}\right)} \cap \partial \Omega=\{\xi\},
$$

where $\overline{B_{\rho}\left(x_{0}\right)}$ denotes the closure of $B_{\rho}\left(x_{0}\right)$.

Proposition 4.9 (Positivity of the solution near the boundary) Assume $u_{0}>0$ in $\Omega$. Then every nonnegative weak supersolution u to (3.1') is positive near the boundary.

Proof We will follow the similar idea as [23]. Since, $\Omega$ satisfies the interior ball condition, we have, for every boundary point $\xi \in \partial \Omega$,

$$
\overline{B_{\rho}\left(x_{0}\right)} \cap \partial \Omega=\{\xi\} .
$$

Take $\rho^{\prime} \in(0, \rho)$ and define the annulus

$$
A:=B_{\rho}\left(x_{0}\right) \backslash \overline{B_{\rho^{\prime}}\left(x_{0}\right)} .
$$




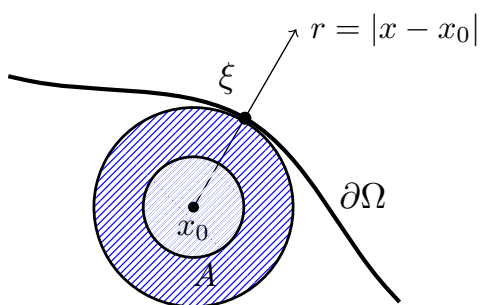

Figure: Interior ball condition

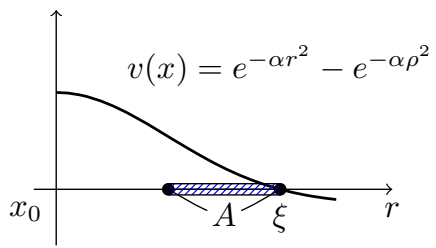

Figure: Graph of $v(x)$

We define a function $v$ for $(x, t) \in A \times[0, T]$ as

$$
v(x, t)=v(x):=e^{-\alpha r^{2}}-e^{-\alpha \rho^{2}},
$$

where $r:=\left|x-x_{0}\right|<\rho$ and $\alpha>0$ is to be determined later. Since

$$
\begin{cases}v_{x_{j}}=-2 \alpha\left(x_{j}-x_{0, j}\right) e^{-\alpha r^{2}}, & j=1, \ldots, n \\ v_{x_{i} x_{j}}=-2 \alpha \delta_{i j} e^{-\alpha r^{2}}+4 \alpha^{2}\left(x_{i}-x_{0, i}\right)\left(x_{j}-x_{0, j}\right) e^{-\alpha r^{2}}, & i, j=1, \ldots, n,\end{cases}
$$

we have

$$
\begin{aligned}
\Delta_{p} v= & (p-2)|\nabla v|^{p-4} e^{-3 \alpha r^{2}} 8 \alpha^{3} r^{2}(-1+2 \alpha r) \\
& +|\nabla v|^{p-2} e^{-\alpha r^{2}} 2 \alpha\left(-n+2 \alpha r^{2}\right)
\end{aligned}
$$

and thus, we can choose a sufficiently large $\alpha$ so that

$$
\Delta_{p} v(x, t) \geq 0 \text { in } A \times[0, T],
$$

where $\alpha$ is chosen depending on $\rho$ and $\rho^{\prime}$. Therefore, by $\partial_{t} v^{q}=0$

$$
\partial_{t} v^{q}-\Delta_{p} v \leq 0 \text { in } A \times[0, T] .
$$

Now, let $m:=\min \left\{\min _{\Omega} u_{0}, \min _{\partial B_{\rho^{\prime}}\left(x_{0}\right) \times[0, T]} u\right\}$. We will show that $m v(x, t)$ is a lower comparison function for the solution. We note that the solution $u$ is uniformly (Hölder) continuous in $\Omega_{T}=\Omega \times(0, T)$ (see Sect. 5.2 below), again, we can choose $\alpha>0$ to be so large that, on the initial boundary $A \times\{t=0\}$,

$$
u(x, 0)=u_{0}(x) \geq m v(x, 0)=m\left(e^{-\alpha r^{2}}-e^{-\alpha \rho^{2}}\right) .
$$

Also,

$$
u(x, t) \geq m v(x, t) \quad \text { on } \partial B_{\rho}\left(x_{0}\right) \times(0, T),
$$


since, on $\partial B_{\rho}\left(x_{0}\right) \times[0, T], v=0$ by definition and $u \geq 0$ by Corollary 4.6. On $\partial B_{\rho^{\prime}}\left(x_{0}\right) \times[0, T]$, by the very definition of $m$,

$$
u(x, t) \geq m\left(e^{-\alpha \rho^{\prime 2}}-e^{-\alpha \rho^{2}}\right)=m v(x, t) \quad \text { on } \partial B_{\rho^{\prime}}\left(x_{0}\right) \times(0, T)
$$

From (4.56), (4.57) and (4.58), we find that

$$
u \geq m v \text { on } \partial_{p} A_{T}
$$

where $A_{T}:=A \times(0, T)$ and $\partial_{p} A_{T}$ is the parabolic boundary of $A_{T}$ and thus, we have that $m v(x, t)$ is lower comparison function for $u$ in $A_{T}=A \times(0, T)$. By Theorem 3.6 , we arrive at

$$
u \geq m v>0 \text { in } A_{T} .
$$

Thus the assertion is actually verified.

\section{The $p$-Sobolev Flow}

\subsection{Positivity of the $p$-Sobolev Flow}

In what follows, we consider the $p$-Sobolev flow (1.1). We first notice the nonnegativity of a solution of the $p$-Sobolev flow.

Proposition 5.1 (Nonnegativity of the $p$-Sobolev flow) Suppose $u_{0} \geq 0$ in $\Omega$. Then, a weak solution $u$ of (1.1) satisfies

$$
u \geq 0 \text { in } \Omega_{T} .
$$

Proof Let $0<t_{1}<t \leq T$ be arbitrarily taken and fixed. Let $\sigma_{t_{1}, t}$ be the same Lipschitz cut-off function on time as in the proof of Proposition 3.4. The function $-(-u)_{+} \sigma_{t_{1}, t}$ is an admissible test function in (D2) of Definition 3.2, since $\partial_{t}\left(|u|^{q-1} u\right) \in L^{2}\left(\Omega_{T}\right)$ by (D1) of Defintion 3.2 and, $-(-u)_{+} \sigma_{t_{1}, t}$ is in $L^{q+1}\left(\Omega \times\left(t_{1}, t\right)\right)$. Thus, we have

$$
\begin{aligned}
& \int_{\Omega_{t_{1}, t}} \partial_{\tau}\left(|u|^{q-1}(-u)\right)(-u)_{+} \sigma_{t_{1}, t} \mathrm{~d} z \\
& \quad+\int_{\Omega_{t_{1}, t}}|\nabla u|^{p-2} \nabla(-u) \cdot \nabla\left((-u)_{+} \sigma_{t_{1}, t}\right) \mathrm{d} z \\
& =\int_{\Omega_{t_{1}, t}} \lambda(\tau)|u|^{q-1}(-u)(-u)_{+} \sigma_{t_{1}, t} \mathrm{~d} z .
\end{aligned}
$$

Applying the very same argument as in the proof of Proposition 3.4 to (5.2), we obtain that

$$
\frac{q}{q+1} \int_{\Omega}(-u(t))_{+}^{q+1} \mathrm{~d} x \leq \int_{0}^{t}(\lambda(\tau))_{+} \int_{\Omega}(-u(\tau))_{+}^{q+1} \mathrm{~d} x \mathrm{~d} \tau .
$$


From the Gronwall lemma it follows that

$$
\int_{\Omega}(-u(t))_{+}^{q+1} \mathrm{~d} x \leq 0
$$

since (D4) in Definition 3.2, $(-u(t))_{+} \rightarrow 0$ in $L^{q+1}(\Omega)$ as $t \searrow 0$. Therefore we have $-u(x, t) \leq 0$ for $(x, t) \in \Omega_{T}$ and the claim is actually verified.

We now state the fundamental energy estimate.

Proposition 5.2 (Energy equality) Let u be a nonnegative weak solution to (1.1). Then the following identities are valid:

(i)

$$
\lambda(t)=\int_{\Omega}|\nabla u(x, t)|^{p} \mathrm{~d} x, \quad t \in[0, T]
$$

(ii)

$$
q \int_{\Omega_{0, t}} u^{q-1}\left(\partial_{t} u\right)^{2} \mathrm{~d} z+\frac{1}{p} \lambda(t)=\frac{1}{p} \lambda(0), \quad t \in[0, T] .
$$

In particular,

$$
\lambda(t) \leq \lambda(0), \quad t \in[0, T]
$$

The proof of this proposition is postponed, and will be given in Appendix B.

Proposition 5.3 (Boundedness of the $p$-Sobolev flow) Let $u \geq 0$ be a weak solution of the p-Sobolev flow equation (1.1). Then $u$ is bounded from above in $\Omega_{T}$ and

$$
\left\|(u(t))_{+}\right\|_{L^{\infty}(\Omega)} \leq e^{\lambda(0) T / q}\left\|u_{0}\right\|_{L^{\infty}(\Omega)} .
$$

Proof By (5.3) we have that $\lambda(t) \leq \lambda(0)$. Therefore, $u$ is a weak subsolution of (3.1) with $M=\left\|u_{0}\right\|_{L^{\infty}(\Omega)}$ and $c=\lambda(0)$. The result then follows by Proposition 3.5.

In general, the solution to (3.1') may vanish at a finite time, however, under the volume constraint as in (1.1), the solution may positively expand in all of times (see Corollary 4.8). This is actually the assertion of the following proposition.

Proposition 5.4 (Interior positivity by the volume constraint) Let $\Omega^{\prime}$ be a subdomain compactly contained in $\Omega$ and very close to $\Omega$. Let $T$ be any positive number and assume that $u_{0}>0$ in $\Omega$. Let $u$ be a nonnegative weak solution of (1.1). Then there exists a positive constant $\bar{\eta}$ such that

$$
u(x, t) \geq \bar{\eta} \text { in } \Omega^{\prime} \times[0, \infty) .
$$

Proof of Proposition 5.4 By the volume constraint together with Proposition 5.3, letting $M:=e^{\lambda(0) T / q}\left\|u_{0}\right\|_{L^{\infty}(\Omega)}$, we have, for a positive number $L<M$ and any $t \in[0, \infty)$ 


$$
\begin{aligned}
1= & \int_{\Omega} u^{q+1}(t) \mathrm{d} x=\int_{\Omega^{\prime} \cap\{u(t) \geq L\}} u^{q+1}(t) \mathrm{d} x+\int_{\Omega^{\prime} \cap\{u(t)<L\}} u^{q+1}(t) \mathrm{d} x \\
& +\int_{\Omega \backslash \Omega^{\prime}} u^{q+1}(t) \mathrm{d} x \\
\leq & M^{q+1}\left|\Omega^{\prime} \cap\{u(t) \geq L\}\right|+L^{q+1}\left|\Omega^{\prime}\right|+M^{q+1}\left|\Omega \backslash \Omega^{\prime}\right| ;
\end{aligned}
$$

i.e.,

$$
\frac{1-L^{q+1}\left|\Omega^{\prime}\right|-M^{q+1}\left|\Omega \backslash \Omega^{\prime}\right|}{M^{q+1}} \leq\left|\Omega^{\prime} \cap\{u(t) \geq L\}\right| .
$$

Choose $\Omega^{\prime}$ such that $\left|\Omega \backslash \Omega^{\prime}\right| \leq \frac{1}{4 M^{q+1}}$ and $L>0$ satisfying $L^{q+1}\left|\Omega^{\prime}\right| \leq \frac{1}{4}$. Under such choice of $\Omega^{\prime}$ and $L$, we find that, for any $t \in(0, \infty)$,

$$
\alpha\left|\Omega^{\prime}\right| \leq\left|\Omega^{\prime} \cap\{u(t) \geq L\}\right|
$$

where $\alpha:=\frac{1}{2 M^{q+1}\left|\Omega^{\prime}\right|}$. Using (5.3), for a nonnegative weak solution $u$ of (1.1), we see that $u$ is a weak supersolution to (3.1') with $c=\lambda(T)$. Thus, from Proposition 4.7, there exist positive integer $N=N\left(\Omega^{\prime}\right)$ and positive number families $\left\{\delta_{m}\right\}_{m=0}^{N},\left\{\eta_{m}\right\}_{m=1}^{N+1} \subset(0,1),\left\{J_{m}\right\}_{m=0}^{N},\left\{I_{m}\right\}_{m=0}^{N} \subset \mathbb{N}$ depending on $p, n, \alpha$ and independent of $L$, a time $t_{N}>t$ such that, for any $t \in[0, \infty)$,

$$
u \geq \eta_{N+1} L \quad \text { a.e. in } \Omega^{\prime} \times \mathcal{I}_{N}^{(k)}(t)
$$

where

$\mathcal{I}_{N}^{(k)}(t):=\left(t_{N}+\left(k+\frac{1}{2}\right) \frac{\delta_{N}\left(\eta_{N} L\right)^{q+1-p}}{2^{J_{N}+I_{N}}} \rho^{p}, t_{N}+(k+1) \frac{\delta_{N}\left(\eta_{N} L\right)^{q+1-p}}{2^{J_{N}+I_{N}}} \rho^{p}\right)$

for some $k \in\left\{0,1, \ldots, 2^{J_{N}+I_{N}}-1\right\}, 0<\rho \leq \operatorname{dist}\left(\Omega^{\prime}, \partial \Omega\right) / 4$ and $t_{N}$ is written as

$$
t_{N}=t+\sum_{m=1}^{N}\left(\ell+\frac{3}{4}\right) \frac{\delta_{m-1}\left(\eta_{m-1} L\right)^{q+1-p}}{2^{J_{m-1}+I_{m-1}}} \rho^{p}
$$

for some $\ell \in\left\{0,1, \ldots, 2^{J_{m-1}+I_{m-1}}-1\right\}$. On the other hand, from $u_{0}>0$ in $\Omega$ and Corollary 4.8, there exist positive numbers $\eta$ and $\tau$ such that

$$
u \geq \eta \quad \text { a.e. in } \Omega^{\prime} \times(0, \tau)
$$

Here we can choose that $\mathcal{I}_{N}^{(k)}(0) \subset(0, \tau)$ from the proof of Proposition 4.7 and Corollary 4.8. Since $t \in[0, \infty)$ is arbitrarily taken, letting $\bar{\eta}:=\min \left\{\eta, \eta_{N+1} L\right\}$, we have that

$$
u(x, t)>\bar{\eta} \text { a.e. in } \Omega^{\prime} \times[0, \infty)
$$


which is our assertion of Proposition 5.4.

Proposition 5.5 (Positivity around the boundary for $p$-Sobolev flow) Suppose that $u_{0}>0$ in $\Omega$. Let $u$ be any nonnegative weak solution to (1.1). Then $u$ is positive near the boundary.

Proof Since a nonegative weak solution of (1.1) is a nonnegative weak supersolution of (3.1') in $\Omega_{\infty}$ with $c=0$, we can apply the proof of Proposition 4.9. Thus the proof is complete.

\subsection{Hölder and Gradient Hölder Continuity}

In this section, we will prove the Hölder and gradient Hölder continuity of the solution to $p$-Sobolev flow (1.1) with respect to space-time variable.

Suppose $u_{0}>0$ in $\Omega$. Then by Propositions 5.4 and 5.3, for any $\Omega^{\prime}$ compactly contained in $\Omega$ and $T \in(0, \infty)$, we can choose a positive constant $\tilde{c}$ such that

$$
0<\tilde{c} \leq u \leq M=: e^{\lambda(0) T / q}\left\|u_{0}\right\|_{L^{\infty}(\Omega)} \quad \text { in } \quad \Omega^{\prime} \times[0, T] .
$$

Under such positivity of a solution in the domain as in (5.5), we can rewrite the first equation of (1.1) as follows : Set $v:=u^{q}$, which is equivalent to $u=v^{\frac{1}{q}}$ and put $g:=\frac{1}{q} v^{1 / q-1}$ and then, we find that the first equation of (1.1) is equivalent to

$$
\partial_{t} v-\operatorname{div}\left(|\nabla v|^{p-2} g^{p-1} \nabla v\right)=\lambda(t) v \quad \text { in } \quad \Omega^{\prime} \times[0, T]
$$

and thus, $v$ is a positive and bounded weak solution of the evolutionary $p$-Laplacian equation (5.6). By (5.5) $g$ is uniformly elliptic and bounded in $\Omega_{T}^{\prime}$. Then we have a local energy inequality for a local weak solution $v$ to (5.6) in Appendix C.1 (see [6]).

The following Hölder continuity is proved via using the local energy inequality, Lemma C.1 in Appendix C.1 and standard iterative real analysis methods. See [6, Chap. III] or [26, Sect.4.4, pp. 44-47] for more details.

Theorem 5.6 (Hölder continuity) Let $v$ be a positive and bounded weak solution to (5.6). Then $v$ is Hölder continuous in $\Omega_{T}^{\prime}$ with a Hölder exponent $\beta \in(0,1)$ on a parabolic metric $|x|+|t|^{1 / p}$.

By a positivity and boundedness as in (5.5) and a Hölder continuity in Theorem 5.6, we see that the coefficient $g^{p-1}$ is Hölder continuous and thus, obtain a Hölder continuity of its spacial gradient.

Theorem 5.7 (Gradient interior Hölder continuity) Let $v$ be a positive and bounded weak solution to (5.6). Then, there exist a positive exponent $\alpha<1$ depending only on $n, p, \beta$ and a positive constant $C$ depending only on $n, p, \tilde{c}, M, \lambda(0), \beta,\|\nabla v\|_{L^{p}\left(\Omega_{T}^{\prime}\right)}$, $[g]_{\beta, \Omega_{T}^{\prime}}$ and $[v]_{\beta, \Omega_{T}^{\prime}}$ such that $\nabla v$ is Hölder continuous in $\Omega_{T}^{\prime}$ with an exponent $\alpha$ on the usual parabolic distance. Furthermore, its Hölder constant is bounded above by $C$, where $[f]_{\beta}$ denotes the Hölder semi-norm of a Hölder continuous function $f$ with a Hölder exponent $\beta$. 
The outline of proof of Theorem 5.7 is presented in Appendix C.

By an elementary algebraic estimate and an interior positivity, boundedness and a Hölder regularity of $v$ and its gradient $\nabla v$ in Theorems 5.6 and 5.7, we also have a Hölder regularity of the solution $u$ and its gradient $\nabla u$.

Theorem 5.8 (Hölder and Gradient Hölder continuity of solutions to the $p$-Sobolev flow) Let $u$ be a positive and bounded weak solution to the p-Sobolev flow (1.1). Then, there exist a positive exponent $\gamma<1$ depending only on $n, p, \beta, \alpha$ and a positive constant $C$ depending only on $n, p, \tilde{c}, M, \lambda(0), \beta, \alpha,\|\nabla u\|_{L^{p}\left(\Omega_{T}^{\prime}\right)},[g]_{\beta, \Omega_{T}^{\prime}}$ and $[v]_{\beta, \Omega_{T}^{\prime}}$ such that $u$ and $\nabla u$ is Hölder continuous in $\Omega_{T}^{\prime}$ with an exponent $\gamma$ on a parabolic metric $|x|+|t|^{1 / p}$ and on the parabolic one, respectively. The Hölder constants are bounded above by $C$, where $[f]_{\beta}$ denotes the Hölder semi-norm of a Hölder continuous function $f$ with a Hölder exponent $\beta$.

Acknowledgements Open access funding provided by University of Helsinki including Helsinki University Central Hospital. This project has received funding from the Academy of Finland and the European Research Council (ERC) under the European Union's Horizon 2020 research and innovation programme (grant agreement No 818437).

Open Access This article is licensed under a Creative Commons Attribution 4.0 International License, which permits use, sharing, adaptation, distribution and reproduction in any medium or format, as long as you give appropriate credit to the original author(s) and the source, provide a link to the Creative Commons licence, and indicate if changes were made. The images or other third party material in this article are included in the article's Creative Commons licence, unless indicated otherwise in a credit line to the material. If material is not included in the article's Creative Commons licence and your intended use is not permitted by statutory regulation or exceeds the permitted use, you will need to obtain permission directly from the copyright holder. To view a copy of this licence, visit http://creativecommons.org/licenses/by/4.0/.

\section{Appendix A: Some Fundamental Facts}

\section{A.1: $L^{2}$ Estimate of the Time Derivative}

We will show the existence in $L^{2}\left(\Omega_{T}\right)$ of time-derivative for a weak solution to (1.1).

Lemma A.1 Let $u$ be a nonnegative solution to (1.1).Then there exists $\partial_{t} u$ in a weak sense, such that $\partial_{t} u \in L^{2}\left(\Omega_{T}\right)$.

Proof of Lemma A.1 Let $a>0$ and $\varepsilon>0$ be arbitrary given. Let $u \geq 0$ be a weak solution to (1.1). Let us define a truncated Lipschitz function $\phi_{\varepsilon}(x)$ by

$$
\phi_{\varepsilon}(x):= \begin{cases}0 & (0 \leq x \leq a) \\ \frac{1}{\varepsilon}(x-a) & (a \leq x \leq a+\varepsilon), \\ 1 & (x \geq a+\varepsilon)\end{cases}
$$

We also set $h(v):=v^{1 / q}$ for $v \geq 0$. For any $\varphi \in C_{0}^{\infty}\left(\Omega_{T}\right)$,

$$
\int_{\Omega_{T}} u \phi_{\varepsilon}(u) \partial_{t} \varphi \mathrm{d} z=-\int_{\Omega_{T}} \partial_{t}\left(u \phi_{\varepsilon}(u)\right) \varphi \mathrm{d} z=-\int_{\Omega_{T}} \partial_{t}\left(\left(u^{q}\right)^{1 / q} \phi_{\varepsilon}(u)\right) \varphi \mathrm{d} z
$$




$$
\begin{aligned}
& =-\int_{\Omega_{T}}\left[\partial_{t} h\left(u^{q}\right) \phi_{\varepsilon}(u)+u \partial_{t} \phi_{\varepsilon}(u)\right] \varphi \mathrm{d} z \\
& =-\int_{\Omega_{T}}\left[\partial_{t} h\left(u^{q}\right) \phi_{\varepsilon}(u)+u \frac{1}{\varepsilon} \chi_{\{a \leq u \leq a+\varepsilon\}} \partial_{t}\left(\left(u^{q}\right)^{1 / q}\right)\right] \varphi \mathrm{d} z \\
& =-\int_{\Omega_{T}} \partial_{t} h\left(u^{q}\right)\left(\phi_{\varepsilon}(u)+u \frac{1}{\varepsilon} \chi_{\{a \leq u \leq a+\varepsilon\}}\right) \varphi \mathrm{d} z .
\end{aligned}
$$

We note that $h(v)=v^{1 / q}$ is locally Lipschitz on $\left\{v=u^{q}: u \geq a\right\}$ and $\partial_{t} u^{q} \in L^{2}\left(\Omega_{T}\right)$ by the very definition (D1) of Definition 3.2 and thus, a composite function $h\left(u^{q}\right)$ is weak differentiable in $\{u \geq a\}$ and

$$
\partial_{t} h\left(u^{q}\right)=h^{\prime}\left(u^{q}\right) \cdot \partial_{t} u^{q} \in L^{2}\left(\Omega_{T} \cap\{u \geq a\}\right),
$$

since $h^{\prime}\left(u^{q}\right)=\frac{1}{q} u^{1-q} \leq \frac{1}{q} a^{1-q}$ on $\{u \geq a\}$. Taking into account of

$$
\begin{aligned}
& \partial_{t} h\left(u^{q}\right) \phi_{\varepsilon}(u) \rightarrow \partial_{t} h\left(u^{q}\right) \chi_{\{u \geq a\}} \quad(\varepsilon \searrow 0), \\
& \left|\partial_{t} h\left(u^{q}\right) \phi_{\varepsilon}(u)\right| \leq \partial_{t} h\left(u^{q}\right) \chi_{\{u \geq a\}} \in L^{1}\left(\Omega_{T}\right)
\end{aligned}
$$

and using the Lebesgue dominated convergence theorem, we have, for the first term on the right hand side of (A.1),

$$
-\lim _{\varepsilon \searrow 0} \int_{\Omega_{T}} \partial_{t} h\left(u^{q}\right) \phi_{\varepsilon}(u) \varphi \mathrm{d} z=-\int_{\Omega_{T}} \partial_{t} h\left(u^{q}\right) \chi_{\{u \geq a\}} \varphi \mathrm{d} z .
$$

By Lemma A.2 the second term on the right hand side of (A.1) is computed as

$$
\lim _{\varepsilon \searrow 0} \int_{\Omega_{T}} \partial_{t} h\left(u^{q}\right) \frac{u}{\varepsilon} \chi_{\{a \leq u \leq a+\varepsilon\}} \varphi \mathrm{d} z=a \int_{\Omega_{T} \cap\{u=a\}} \partial_{t} h\left(u^{q}\right) \varphi \mathrm{d} z .
$$

By Lebesgue's dominated convergence theorem, the left hand side of (A.1) is computed as

$$
\int_{\Omega_{T}} u \phi_{\varepsilon}(u) \partial_{t} \varphi \mathrm{d} z \rightarrow \int_{\Omega_{T}} u \chi_{\{u \geq a\}} \partial_{t} \varphi \mathrm{d} z .
$$

Passing to the limit as $\varepsilon \searrow 0$ in (A.1) and gathering (A.2), (A.3) and (A.4), we have

$$
\int_{\Omega_{T}} u \chi_{\{u \geq a\}} \partial_{t} \varphi \mathrm{d} z=-\int_{\Omega_{T}} \partial_{t} h\left(u^{q}\right) \chi_{\{u \geq a\}} \varphi \mathrm{d} z+a \int_{\Omega_{T} \cap\{u=a\}} \partial_{t} h\left(u^{q}\right) \varphi \mathrm{d} z .
$$

Again, by Lebesgue's dominated theorem, taking the limit as $a \searrow 0$ in the above formula, we have

$$
\int_{\Omega_{T}} u \partial_{t} \varphi \mathrm{d} z=-\int_{\Omega_{T}} \partial_{t} h\left(u^{q}\right) \varphi \mathrm{d} z=-\int_{\Omega_{T}} \partial_{t} u \cdot \varphi \mathrm{d} z,
$$

which completes the proof. 
In the proof above, we used the following lemma as for the convergence of Dirac measure.

Lemma A.2 Let $a>0$ and $\varepsilon>0$ be arbitrary given. Then

$$
\frac{1}{\varepsilon} \chi_{\{a \leq u \leq a+\varepsilon\}} \rightarrow \delta_{(a)} \text { as } \varepsilon \searrow 0 \text { in } \mathscr{D}^{\prime}(\mathbb{R})
$$

where we denote by $\mathscr{D}^{\prime}(\mathbb{R})$ the distribution function space, which is the dual space of the space $\mathscr{D}$ of smooth functions with compact support in $\mathbb{R}$.

Proof For any $\psi \in \mathscr{D}$, we have

$$
\int_{-\infty}^{\infty} \frac{1}{\varepsilon} \chi_{\{a \leq u \leq a+\varepsilon\}} \psi \mathrm{d} u=\frac{1}{\varepsilon} \int_{a}^{a+\varepsilon} \psi \mathrm{d} u \rightarrow \psi(a)=\left\langle\delta_{(a)}, \psi\right\rangle
$$

as $\varepsilon \searrow 0$. Therefore we have for any $\psi \in \mathscr{D}$

$$
\lim _{\varepsilon \searrow 0}\left\langle\frac{1}{\varepsilon} \chi_{\{a \leq u \leq a+\varepsilon\}}, \psi\right\rangle=\left\langle\delta_{(a)}, \psi\right\rangle,
$$

which is our claim.

\section{A.2: Regularization}

In this subsection, we will show the following regularization, Lemma A.3. Before stating assertion, we prepare some notations. For $f \in L_{l o c}^{1}\left(\Omega_{T}\right)$, we denote the mollifier of $f$ by

$$
f_{\varepsilon, h}(z):=\int_{Q_{\varepsilon, h}(z)} \rho_{\varepsilon, h}\left(z^{\prime}-z\right) f\left(z^{\prime}\right) \mathrm{d} z^{\prime} .
$$

Here $\varepsilon>0, z=(x, t), Q_{\varepsilon, h}(z)=B_{\varepsilon}(x) \times(t-h, t+h) \subset \Omega_{T}$, and

$$
\rho_{\varepsilon, h}(z):=\frac{1}{h} \rho_{1}\left(\frac{t}{h}\right) \frac{1}{\varepsilon^{n}} \rho_{2}\left(\frac{x}{\varepsilon}\right)
$$

where $\rho_{1}$ and $\rho_{2}$ are smooth symmetric in the following sense:

$$
\rho_{1}(t)=\rho_{1}(-t), \quad \rho_{2}(x)=\rho_{2}(|x|),
$$

and satisfies

$$
\operatorname{supp}\left(\rho_{1}\right) \subset(-1,1), \int_{\mathbb{R}} \rho_{1}(t) \mathrm{d} t=1 ; \quad \operatorname{supp}\left(\rho_{2}\right) \subset B_{1}(0), \quad \int_{\mathbb{R}^{n}} \rho_{2}(x) d x=1 .
$$

Lemma A.3 Let $0<t_{1}<t<T$. For the weak solution $u$ to (3.1'),

$$
\left.\int_{\Omega_{t_{1}, t}}|\nabla u|^{p-2} \nabla u \cdot \nabla\left(\left(\partial_{t} u\right)_{\varepsilon, h}\right)_{\varepsilon,-h} \mathrm{~d} z \longrightarrow \int_{\Omega} \frac{1}{p}|\nabla u|^{p} \mathrm{~d} x\right|_{t_{1}} ^{t} \text { as } \varepsilon, h \searrow 0 .
$$


Proof From (D1) in Definition 3.2 and Fubini's theorem, it follows that

$$
\begin{aligned}
& \int_{\Omega_{t_{1}, t}}|\nabla u|^{p-2} \nabla u \cdot \nabla\left(\left(\partial_{t} u\right)_{\varepsilon, h}\right)_{\varepsilon,-h} \mathrm{~d} z \\
& =\int_{\Omega_{t_{1}, t}}\left(|\nabla u|^{p-2} \nabla u\right)_{\varepsilon, h} \cdot \nabla\left(\partial_{t} u\right)_{\varepsilon, h} \mathrm{~d} z \\
& =-\int_{\Omega_{t_{1}, t}}\left[\operatorname{div}\left(|\nabla u|^{p-2} \nabla u\right)_{\varepsilon, h}-\operatorname{div}\left(\left|\nabla u_{\varepsilon, h}\right|^{p-2} \nabla u_{\varepsilon, h}\right)\right] \partial_{t} u_{\varepsilon, h} \mathrm{~d} z \\
& \quad+\int_{\Omega_{t_{1}, t}}\left|\nabla u_{\varepsilon, h}\right|^{p-2} \nabla u_{\varepsilon, h} \cdot \partial_{t}\left(\nabla u_{\varepsilon, h}\right) \mathrm{d} z \\
& =: I+J .
\end{aligned}
$$

Since $\operatorname{div}\left(|\nabla u|^{p-2} \nabla u\right) \in L^{2}\left(\Omega_{T}\right)$ by (D1) and (D2) again, we have, as $\varepsilon, h \searrow 0$,

$$
\begin{aligned}
& \left(\operatorname{div}\left(|\nabla u|^{p-2} \nabla u\right)\right)_{\varepsilon, h}, \operatorname{div}\left(\left|\nabla u_{\varepsilon, h}\right|^{p-2} \nabla u_{\varepsilon, h}\right) \rightarrow \operatorname{div}\left(|\nabla u|^{p-2} \nabla u\right) \\
& \text { strongly in } L^{2}\left(\Omega_{T}\right)
\end{aligned}
$$

and, by Lemma A.1, as $\varepsilon, h \searrow 0$,

$$
\left(\partial_{t} u\right)_{\varepsilon, h} \rightarrow \partial_{t} u \text { strongly in } L^{2}\left(\Omega_{T}\right)
$$

It follows from (A.6) and (A.7) that

$$
I \rightarrow 0 \text { as } \varepsilon, h \searrow 0 .
$$

By (D1) in Definition 3.2 we have, as $\varepsilon, h \rightarrow 0$,

$$
J=\left.\left.\int_{\Omega} \frac{1}{p}\left|\nabla u_{\varepsilon, h}\right|^{p} \mathrm{~d} x\right|_{t_{1}} ^{t} \rightarrow \int_{\Omega} \frac{1}{p}|\nabla u|^{p} \mathrm{~d} x\right|_{t_{1}} ^{t}
$$

and thus, gather (A.8), (A.9) and (A.5) to complete the proof.

\section{Appendix B: Proof of Proposition 5.2}

This section is devoted to prove Proposition 5.2.

Proof of Proposition 5.2 (i) Let $0<t_{1}<t<T$ be arbitrarily taken and let $\sigma_{t_{1}, t}$ be the same time cut-off function as in the proof of Proposition 3.4. The function $\sigma_{t_{1}, t} u$ is in $L^{\infty}\left(t_{1}, t ; W^{1, p}(\Omega)\right)$, and nonnegative by Proposition 5.1 and thus, is an admissible test function in (D2) for (1.1). Choose a test function as $\sigma_{t_{1}, t} u$ in (D2) for (1.1), to have 


$$
\int_{\Omega_{t_{1}, t}} \partial_{\tau}\left(u^{q}\right) \sigma_{t_{1}, t} u \mathrm{~d} z+\int_{\Omega_{t_{1}, t}}|\nabla u|^{p-2} \nabla u \cdot \nabla\left(\sigma_{t_{1}, t} u\right) \mathrm{d} z=\int_{\Omega_{t_{1}, t}} \lambda(\tau) u^{q} \sigma_{t_{1}, t} u \mathrm{~d} z .
$$

By the very definition of $A^{+}(u)$ for $u \geq 0$, the first term on the left hand side of (B.1) is computed as

$$
\begin{aligned}
\int_{\Omega_{t_{1}, t}} \partial_{\tau}\left(u^{q}\right) \sigma_{t_{1}, t} u \mathrm{~d} z & =\int_{\Omega_{t_{1}, t}} \partial_{\tau} A^{+}(u) \sigma_{t_{1}, t} \mathrm{~d} z \\
& =\left.\int_{\Omega} A^{+}(u) \sigma_{t_{1}, t} \mathrm{~d} x\right|_{t_{1}} ^{t}-\int_{\Omega_{t_{1}, t}} A^{+}(u) \partial_{\tau} \sigma_{t_{1}, t} \mathrm{~d} z \\
& \rightarrow \int_{\Omega} \frac{q}{q+1} u(t)^{q+1} \mathrm{~d} x-\int_{\Omega} \frac{q}{q+1} u\left(t_{1}\right)^{q+1} \mathrm{~d} x \quad \text { as } \quad \delta \searrow 0 .
\end{aligned}
$$

The second term on the left hand side of (B.1) is treated as

$$
\int_{\Omega_{t_{1}, t}}|\nabla u|^{p-2} \nabla u \cdot \nabla\left(\sigma_{t_{1}, t} u\right) \mathrm{d} z=\int_{\Omega_{t_{1}, t}}|\nabla u|^{p} \sigma_{t_{1}, t} \mathrm{~d} z \rightarrow \int_{\Omega_{t_{1}, t}}|\nabla u|^{p} \mathrm{~d} z \quad \text { as } \delta \searrow 0 .
$$

Using (B.2), (B.3) and the volume preserving condition $\int_{\Omega} u(x, t)^{q+1} \mathrm{~d} x=1, t \geq 0$, we take the limit as $\delta \searrow 0$ in (B.1) to obtain that

$$
\int_{\Omega_{t_{1}, t}}|\nabla u|^{p} \mathrm{~d} z=\int_{\Omega_{t_{1}, t}} \lambda(\tau) u^{q+1} \mathrm{~d} z
$$

Dividing above formula by $t-t_{1}$, we have

$$
\frac{1}{t-t_{1}} \int_{t_{1}}^{t} \int_{\Omega}|\nabla u(x, \tau)|^{p} \mathrm{~d} x \mathrm{~d} \tau=\frac{1}{t-t_{1}} \int_{t_{1}}^{t} \int_{\Omega} \lambda(\tau) u^{q+1}(x, \tau) \mathrm{d} x \mathrm{~d} \tau .
$$

According to the volume preserving condition again, passing the limit as $t \searrow t_{1}$ in the formula above, we obtain that

$$
\lambda\left(t_{1}\right)=\int_{\Omega}\left|\nabla u\left(x, t_{1}\right)\right|^{p} \mathrm{~d} x
$$

which is our first assertion.

(ii) We notice the boundedness of the solution $u$ of the p-Sobolev flow. This is shown as follows: By Proposition 5.2 (i) above, $\lambda(t)=\|\nabla u(t)\|_{L^{p}(\Omega)}^{p}$ and thus, $\lambda(t) \in$ $L^{\infty}(0, T)$ by (D1) in Definition 3.2. We also have that $(u)_{+}$is bounded in $\Omega_{T}$ as in Proposition 5.3, and thus, $u$ itself bounded by Proposition 5.1. Consequently, the function $\sigma_{t_{1}, t} \partial_{t} u$ is an admissible test function in (D2) of Definition 3.2 by Lemmata A.1 and A.3. We now take a test function as $\sigma_{t_{1}, t} \partial_{t} u$ in (D2) of Definition 3.2 and then 


$$
\begin{aligned}
& \int_{\Omega_{t_{1}, t}} \partial_{t}\left(u^{q}\right) \sigma_{t_{1}, t} \partial_{t} u \mathrm{~d} z+\int_{\Omega_{t_{1}, t}}|\nabla u|^{p-2} \nabla u \cdot \nabla\left(\sigma_{t_{1}, t} \partial_{t} u\right) \mathrm{d} z \\
& \quad=\int_{\Omega_{t_{1}, t}} \lambda(t) u^{q} \sigma_{t_{1}, t} \partial_{t} u \mathrm{~d} z,
\end{aligned}
$$

Note that the integral on the right hand side in (B.4) is finite by Proposition 3.5 and Lemma A.1. Using the Lebesgue dominated theorem with Proposition 3.5 and Lemma A.1, the first term on the left hand side of (B.4) is computed as

$$
\begin{aligned}
\int_{\Omega_{t_{1}, t}} \partial_{t} u^{q} \sigma_{t_{1}, t} \partial_{t} u \mathrm{~d} z & =q \int_{\Omega_{t_{1}, t}} u^{q-1}\left(\partial_{t} u\right)^{2} \sigma_{t_{1}, t} \mathrm{~d} z \\
& \rightarrow q \int_{\Omega_{t_{1}, t}} u^{q-1}\left(\partial_{t} u\right)^{2} \mathrm{~d} z \text { as } \delta \searrow 0 .
\end{aligned}
$$

The second term on the left hand side of (B.4) is treated as

$$
\begin{aligned}
& \int_{\Omega_{t_{1}, t}}|\nabla u|^{p-2} \nabla u \cdot \nabla\left(\sigma_{t_{1}, t} \partial_{t} u\right) \mathrm{d} z \\
& \quad=\int_{\Omega_{t_{1}, t}}|\nabla u|^{p-2} \nabla u \cdot \partial_{t} \nabla u \sigma_{t_{1}, t} \mathrm{~d} z \\
& =\int_{\Omega_{t_{1}, t}} \partial_{t}\left(\frac{1}{p}|\nabla u|^{p}\right) \sigma_{t_{1}, t} \mathrm{~d} z \\
& =\left.\int_{\Omega} \frac{1}{p}|\nabla u|^{p} \sigma_{t_{1}, t} \mathrm{~d} x\right|_{t_{1}} ^{t}-\int_{\Omega_{t_{1}, t}} \frac{1}{p}|\nabla u|^{p} \partial_{t} \sigma_{t_{1}, t} \mathrm{~d} z \\
& \quad \rightarrow \int_{\Omega} \frac{1}{p}|\nabla u(x, t)|^{p} \mathrm{~d} x-\int_{\Omega} \frac{1}{p}\left|\nabla u\left(x, t_{1}\right)\right|^{p} \mathrm{~d} x \quad \text { as } \quad \delta \searrow 0 \\
& =\frac{1}{p} \lambda(t)-\frac{1}{p} \lambda\left(t_{1}\right),
\end{aligned}
$$

where the manipulation in the second and third lines are justified by Lemma A.3 in Appendix A. By the volume conservation $\int_{\Omega} u(x, t)^{q+1}=1, t \geq 0$, the right hand side of (B.4) is calculated as

$$
\int_{\Omega_{t_{1}, t}} \lambda(t) u^{q} \sigma_{t_{1}, t} \partial_{t} u \mathrm{~d} z=\int_{t_{1}}^{t} \lambda(t) \sigma_{t_{1}, t} \frac{\mathrm{d}}{\mathrm{d} t}\left(\int_{\Omega} \frac{u^{q+1}}{q+1} \mathrm{~d} x\right) \mathrm{d} t=0
$$

From (B.5), (B.6) and (B.7), it follows that

$$
q \int_{\Omega_{t_{1}, t}} u^{q-1}\left(\partial_{t} u\right)^{2} \mathrm{~d} z+\frac{1}{p} \lambda(t)-\frac{1}{p} \lambda\left(t_{1}\right)=0 .
$$

Letting $t_{1}=0$, we have the desired result. 


\section{Appendix C: Notes on Hölder Regularity}

\section{C.1: A Local Energy Estimate for (5.6)}

We will derive a local energy estimate for (5.6) here.

Lemma C.1 Let $\theta>0$ be a parameter. For any $z_{0}=\left(x_{0}, t_{0}\right) \in \Omega_{T}^{\prime}$, take $\rho>0$ such that $Q(\theta, \rho)\left(z_{0}\right) \equiv B_{\rho}\left(x_{0}\right) \times\left(t_{0}-\rho^{\theta}, t_{0}\right) \subset \Omega_{T}^{\prime}$. Let $\zeta$ be a piecewise smooth function on $Q(\theta, \rho)\left(z_{0}\right)$ satisfying

$$
0 \leq \zeta \leq 1, \quad|\nabla \zeta|<\infty, \quad \zeta(x, t)=0 \text { outside } Q(\theta, \rho)\left(z_{0}\right)
$$

Furthermore, take a positive number $\delta_{0}$ such that

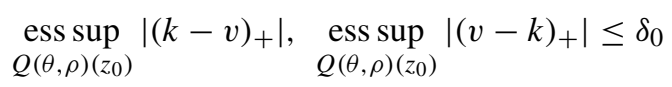

for some $k \geq 0$. Then the following inequality holds true.

(i) Let $v$ be a weak supersolution to (5.6). Then it holds that

$$
\begin{aligned}
& \underset{t_{0}-\rho^{\theta}<t<t_{0}}{\operatorname{ess} \sup _{B_{\rho}\left(x_{0}\right)}}(k-v)_{+}^{2} \zeta^{p} \mathrm{~d} x+\int_{Q(\theta, \rho)\left(z_{0}\right)}\left|\nabla(k-v)_{+}\right|^{p} \zeta^{p} \mathrm{~d} z \\
& \leq \int_{B_{\rho}\left(x_{0}\right) \times\left\{t_{0}-\rho^{\theta}\right\}}(k-v)_{+}^{2} \zeta^{p} \mathrm{~d} x+C \int_{Q(\theta, \rho)\left(z_{0}\right)}(k-v)_{+}^{p}|\nabla \zeta|^{p} \mathrm{~d} z \\
& \quad+\int_{Q(\theta, \rho)\left(z_{0}\right)}(k-v)_{+}^{2} \zeta^{p-1}\left|\zeta_{t}\right| \mathrm{d} z+C \delta_{0} \int_{Q(\theta, \rho)\left(z_{0}\right)} \chi_{\left\{(k-v)_{+}>0\right\}} \mathrm{d} z
\end{aligned}
$$

where $C$ is a positive constant depending only on $n, p, \tilde{c}, M, \lambda(0)$.

(ii) Let $v$ be a weak subsolution to (5.6). Then it holds that

$$
\begin{aligned}
& \underset{t_{0}-\rho^{\theta}<t<t_{0}}{\operatorname{ess} \sup _{B_{\rho}\left(x_{0}\right)}}(v-k)_{+}^{2} \zeta^{p} \mathrm{~d} x+\int_{Q(\theta, \rho)\left(z_{0}\right)}\left|\nabla(v-k)_{+}\right|^{p} \zeta^{p} \mathrm{~d} z \\
& \leq \int_{B_{\rho}\left(x_{0}\right) \times\left\{t_{0}-\rho^{\theta}\right\}}(v-k)_{+}^{2} \zeta^{p} \mathrm{~d} x+C \int_{Q(\theta, \rho)\left(z_{0}\right)}(v-k)_{+}^{p}|\nabla \zeta|^{p} \mathrm{~d} z \\
& \quad+\int_{Q(\theta, \rho)\left(z_{0}\right)}(v-k)_{+}^{2} \zeta^{p-1}\left|\zeta_{t}\right| \mathrm{d} z+C \delta_{0} \int_{Q(\theta, \rho)\left(z_{0}\right)} \chi_{\left\{(v-k)_{+}>0\right\}} \mathrm{d} z
\end{aligned}
$$

where $C$ is a positive constant depending only on $p, n, \tilde{c}, M, \lambda(0)$.

Proof We give the proof only for the case (i). Take a test function as $\varphi=-(k-v)_{+} \zeta^{p}$ in the weak form of (5.6); i.e., 


$$
-\int_{Q_{t}(\theta, \rho)\left(z_{0}\right)} v \partial_{t} \varphi \mathrm{d} z+\int_{Q_{t}(\theta, \rho)\left(z_{0}\right)}|\nabla v|^{p-2} \nabla v \cdot \nabla \varphi \mathrm{d} z=c \int_{Q_{t}(\theta, \rho)\left(z_{0}\right)} v \varphi \mathrm{d} z,
$$

where $Q_{t}(\theta, \rho)\left(z_{0}\right):=B_{\rho}\left(x_{0}\right) \times\left(t_{0}-\rho^{\theta}, t\right)$ for any $t \in\left(t_{0}-\rho^{\theta}, t_{0}\right)$. The first term on the left hand side of (C.3) is computed as

$$
\left.\int_{B_{\rho}\left(x_{0}\right) \times\{t\}} \frac{1}{2}(k-v)_{+}^{2} \zeta^{p} \mathrm{~d} x\right|_{t_{0}-\theta} ^{t}-\int_{Q_{t}(\theta, \rho)} \frac{1}{2}(k-v)_{+}^{2} p \zeta^{p-1} \zeta_{t} \mathrm{~d} z
$$

Meanwhile, by use of (5.5) and Young's inequality, the second term of (C.3) is estimated from below as

$$
\begin{aligned}
& \int_{Q_{t}(\theta, \rho)\left(z_{0}\right)} g^{p-1}|\nabla v|^{p-2} \nabla v \cdot \nabla\left(-(k-v)_{+} \zeta^{p}\right) \mathrm{d} z \\
& \quad=\int_{Q_{t}(\theta, \rho)\left(z_{0}\right)} g^{p-1}\left|\nabla(k-v)_{+}\right|^{p-2} \nabla(k-v)_{+} \cdot \nabla(k-v)_{+} \zeta^{p} \mathrm{~d} z \\
& \quad+\int_{Q_{t}(\theta, \rho)\left(z_{0}\right)} g^{p-1}\left|\nabla(k-v)_{+}\right|^{p-2} \nabla(k-v)_{+} \cdot\left((k-v)_{+} p \zeta^{p-1} \nabla \zeta\right) \mathrm{d} z \\
& \left.\quad \geq c_{0} \int_{Q_{t}(\theta, \rho)\left(z_{0}\right)}\left|\nabla(k-v)_{+}\right|^{p} \zeta^{p} \mathrm{~d} z-c_{1} \int_{Q_{t}(\theta, \rho)\left(z_{0}\right)} \mid k-v\right)_{+}^{p}|\nabla \zeta|^{p} \mathrm{~d} z,
\end{aligned}
$$

where $c_{0}$ and $c_{1}$ are positive constants depending only on $p, n, M$ and $p, n, \tilde{c}$, respectively. By using (5.3) in Proposition 5.2 the right hand side of (C.3) is bounded above by

$$
\left|\lambda(t) \int_{Q_{t}(\theta, \rho)\left(z_{0}\right)} v\left(-(k-v)_{+} \zeta^{p}\right) \mathrm{d} z\right| \leq c_{2} \delta_{0} \int_{Q_{t}(\theta, \rho)\left(z_{0}\right)} \chi_{\left\{(k-v)_{+}>0\right\}} \mathrm{d} z
$$

where $c_{2}$ is a positive constant depending only on $\tilde{c}, \lambda(0)$. Gathering (C.4), (C.5) and (C.6), we arrive at the desired estimate (C.2).

\section{C.2: Outline of Proof of Theorem 5.7}

We recall the outline of proof of Theorem 5.7 here.

Proof By the Hölder continuity in Theorem 5.6, Eq.(5.6) is an evolutionary $p$ Laplacian system with Hölder continuous elliptic and bounded coefficients $g$ and lower order terms $v$. We apply the gradient Hölder regularity for the evolutionary $p$-Laplacian systems with lower order terms in [18, Theorem 1, p. 390] (also see [13]). Here the so-called Campanatto's perturbation method is applied to the gradient Hölder regularity for the evolutionary $p$-Laplacian systems with Hölder coefficients and lower order terms. We also refer to the book in [6, Theorem 1.1, p. 245]. 


\section{References}

1. Alt, H.W., Luckhaus, S.: Quasilinear elliptic-parabolic differential equations. Math. Z. 183, 311-341 (1983)

2. Aubin, T.: Equations différentielles non linéaires et problème de Yamabe concernant la courbure scalaine. J. Math. Pures Appl. 55, 269-296 (1976)

3. Aubin, T.: Some Nonlinear Problems in Riemannian Geometry. Springer Monographs in Mathematics. Springer, Berlin (1997)

4. Avelin, B., Kuusi, T., Nyström, K.: Boundary behavior of solutions to the parabolic p-Laplace equation. Anal. PDE 12(1), 1-42 (2019)

5. Barrett, J.W., Liu, W.B.: Finite element of approximation of the parabolic $p$-Laplacian. SIAM J. Numer. Anal 34(2), 413-428 (1994)

6. DiBenedetto, E.: Degenerate Parabolic Equations. Universitext. Springer, New York (1993)

7. DiBenedetto, E., Gianazza, U., Vespri, V.: Harnack's Inequality for Degenerate and Singular Parabolic Equations. Springer Monographs in Mathematics. Springer, New York (2012)

8. Evans, L.C.: Partial Differential Equations. American Mathematical Society, Providence (1998)

9. Gianazza, U., Vespri, V.: Parabolic De Giorgi classes of order $p$ and the Harnack inequality. Calc. Var. Partial Differ. Equ. 26(3), 379-399 (2006)

10. Hamilton, R.S.: Lectures on Geometric Flows (1989) (unpublished)

11. Ivanov, A.V.: Hölder estimates for a natural class of equations of fast diffusion type Hölder estimates for equations of fast diffusion type. Zap. Nauchn. Sem. S.-Peterburg. Otdel. Mat. Inst. Steklov. (POMI) 229(11), 29-322 (1995). Translation in J. Math. Sci. (New York) 89(6), 1607-1630 (1998)

12. Ivanov, A.V.: Uniform Hölder estimates for generalized solutions of quasilinear parabolic equations that admit double degeneration, Algebra Anal. 3(2), 139-179, 1991. Translation in St. Petersburg Math. J. 3(2), 363-403 (1992)

13. Karim, C., Misawa, M.: Gradient Hölder regularity for nonlinear parabolic systems of p-Laplacian type. Differ. Integr. Equ. 29(3-4), 201-228 (2016)

14. Kinnunen, J., Kuusi, T.: Local behavior of solutions to doubly nonlinear parabolic equations. Math. Ann. 337(3), 705-728 (2007)

15. Kuusi, T.: Harnack estimates for weak supersolutions to nonlinear degenerate parabolic equations. Ann. Sc. Norm. Super. Pisa Cl. Sci. (V) 7, 673-716 (2008)

16. Kuusi, T., Siljander, J., Urbano, J.M.: Local Hölder continuity for doubly nonlinear parabolic equations. Indiana Univ. Math. J. 61(1), 399-430 (2012)

17. Kuusi, T., Mingione, G., Nyström, K.: A boundary Harnack inequality for singular equations of pparabolic type. Proc. Am. Math. Soc. 142(8), 2705-2719 (2014)

18. Misawa, M.: Local Hölder regularity of gradients for evolutional $p$-Laplacian systems. Ann. Mat. Pura Appl. 181, 389-405 (2002)

19. Nakamura, K., Misawa, M.: Existence of a weak solution to the $p$-Sobolev flow. Non. Anal. TMA 175C, 157-172 (2018)

20. Porzio, M.M., Vespri, V.: Hölder estimates for local solutions of some doubly nonlinear degenerate parabolic equations. J. Differ. Equ. 103(1), 146-178 (1993)

21. Schwetlick, H., Struwe, M.: Convergence of the Yamabe flow for large energies. J. Reine Angew. Math. 562, 59-100 (2003)

22. Sciunzi, B.: Classification of positive $\mathcal{D}^{1, p}\left(\mathbb{R}^{N}\right)$-solution to the critical $p$-Laplace equation. Adv. Math. 291, 12-23 (2016)

23. Suzuki, T., Ueoka, Y.: Lecture on Partial Differential Equations-A Course in Semi-linear Elliptic Equations. Baifûkan, Tokyo (2005) (Japanese)

24. Talenti, G.: Best constant in Sobolev inequality. Ann. Mat. Pura Appl. 110(4), 353-372 (1976)

25. Trudinger, N.S.: Pointwise estimates and quasilinear parabolic equations. Commun. Pure Appl. Math. 21, 205-226 (1968)

26. Urbano, J.M.: The Method of Intrinsic Scaling. Lecture Notes in Mathematics, vol. 1930. Springer, Berlin (2008)

27. Vazquez, J.L.: Smoothing and Decay Estimates for Nonlinear Diffusion Equations. Equations of Porous Medium Type. Oxford Lecture Series in Mathematics and Its Applications, vol. 33. Oxford University Press, Oxford (2006)

28. Vazquez, J.L.: The Porous Medium Equation. Mathematical Theory. Oxford Mathematical Monographs. The Clarendon Press, Oxford (2007) 
29. Vespri, V.: On the local behavior of solutions of a certain class of doubly nonlinear parabolic equations. Manuscr. Math. 75, 65-80 (1992)

30. Vespri, V.: Harnack type inequalities for solutions of certain doubly nonlinear parabolic equations. J. Math. Anal. Appl. 181(1), 104-131 (1994)

31. Yamabe, H.: On a deformation of Riemannian structures on compact manifolds. Osaka Math. J. 12, 21-37 (1960)

32. Ye, R.: Global existence and convergence of Yamabe flow. J. Differ. Geom. 39, 35-50 (1994)

Publisher's Note Springer Nature remains neutral with regard to jurisdictional claims in published maps and institutional affiliations. 